\title{
Direct observation of breakdown trigger seeds in a normal-conducting rf accelerating cavity
}

\author{
Tetsuo Abe, Tatsuya Kageyama, Hiroshi Sakai, Yasunao Takeuchi, and Kazuo Yoshino \\ Accelerator Laboratory, High Energy Accelerator Research Organization (KEK), \\ Tsukuba, Ibaraki 305-0801, Japan
}

(Received 17 August 2018; published 26 December 2018)

\begin{abstract}
In our previous study on a normal-conducting 508.9-MHz continuous-wave accelerating cavity, it was discovered that most breakdown events were accompanied by the explosion of a stable bright spot on one of the two end plates of the cavity (Breakdown Type I) or by a spot-type explosion not originating from a stable bright spot (Breakdown Type II); both of these explosions can be understood as breakdown triggers. In the present study, the light emitters of stable bright spots in Breakdown Type I and the dynamics of spot-type explosions in Breakdown Type II were investigated using hyperspectral and high-speed cameras, respectively, during high-power test.
\end{abstract}

DOI: 10.1103/PhysRevAccelBeams.21.122002

\section{INTRODUCTION}

Normal-conducting radio-frequency (rf) accelerating cavities (hereafter simply "cavities"), composed of oxygen-free copper, are at the hearts of many modern particle accelerators. A vacuum arc in a cavity can lead to vacuum breakdown (hereafter called cavity breakdown), which can limit the accelerator performance; however, the actual trigger mechanism of cavity breakdowns (breakdown trigger mechanism) is unknown despite many breakdown studies having been performed by many researchers around the world (e.g., presentations from recent workshops [1,2]).

In this paper, the term "breakdown trigger" is used to refer to a phenomenon that ignites a vacuum arc and is followed by cavity breakdown; the term "breakdown trigger seed" is further defined as a microscopic object or microstructure essential for a breakdown trigger to occur. Finally, the "breakdown trigger mechanism" is the physical process that leads to the occurrence of a breakdown trigger. Therefore, if breakdown trigger seeds are completely removed or the breakdown trigger mechanism is completely blocked, no cavity breakdown occurs.

In 2014, we performed an experimental breakdown study on a normal-conducting $508.9-\mathrm{MHz}$ continuouswave accelerating cavity based on direct in-situ observation [3] guided by the conviction that a "direct" picture is worth

\footnotetext{
*tetsuo.abe@kek.jp
}

Published by the American Physical Society under the terms of the Creative Commons Attribution 4.0 International license. Further distribution of this work must maintain attribution to the author(s) and the published article's title, journal citation, and DOI. a thousand "indirect" measurements. This conviction was inspired by the assumption that breakdown trigger seeds are so minuscule that the information they contain can be mostly destroyed by the much larger phenomenon of vacuum arc. In this previous study, the inside of the cavity was directly observed using three television (TV) cameras during high-power test, and videos were recorded at the moment of cavity breakdowns. From these observations, it was discovered that breakdown events of interest could be classified into two types. The first type is accompanied by the explosion of a stable bright spot on one of the two end plates of the cavity; prior to explosion, this bright spot was observed to maintain its intensity for hours or longer with no significant effects on the accelerating-mode field during high-power operation (Breakdown Type I). No observed breakdown events were accompanied by two or more explosions of stable bright spots; therefore, such an explosion must be a breakdown trigger, and the light emitters of the stable bright spots the corresponding breakdown trigger seeds. The other type was accompanied by a spot-type (i.e., localized) explosion not originating from a stable bright spot (Breakdown Type II). As before, no observed breakdown events were accompanied by two or more such explosions, which means that such explosions must also be breakdown triggers. Breakdown Types I and II constituted approximately $25 \%$ and $40 \%$ of the 205 total breakdown events, respectively. Other events of "pyrotechnical" breakdown, accompanied by a flash or lightning with no observed spot-type explosion, constituted approximately $10 \%$. The remaining events (approximately 25\%) were accompanied by no abnormality observed by any of the three TV cameras at the moment of cavity breakdown. Because breakdown events accompanied by a flash or lightning with no observed spot-type explosion were 
detected only in the initial stage of rf conditioning, essential points of investigation required to elucidate the breakdown trigger mechanism in normal-conducting ultrahigh-frequency continuous-wave cavities are (1) the light emitters of stable bright spots in Breakdown Type I and (2) the dynamics of spot-type explosions in Breakdown Type II.

The focus of the experimental study presented in this paper was the investigation of the above two points using a hyperspectral camera or high-speed cameras for direct insitu observation. This study is a continuation of our previous study [3].

Table $\mathrm{V}$ of Appendix lists the abbreviations used in this paper.

\section{RF ACCELERATING CAVITIES}

For a positron damping ring (DR) at the SuperKEKB accelerator [4], we previously developed normalconducting 508.9-MHz accelerating cavities made of high-purity oxygen-free copper (C10100, ASTM F68 Class 1) with a complete higher-order-mode (HOM) heavily damped structure; this type of cavity is hereafter referred to as the DR cavity [5-8]. Figure 1(a) shows a conceptual diagram of the DR cavity, which is a single-cell pillbox cavity used to excite a continuous $\mathrm{TM}_{010}$ standing wave as the accelerating mode. We fabricated a prototype cavity (DR cavity No. 0) in fiscal year 2011 and performed the first high-power test of this cavity early in fiscal year 2012 at a high-power test stand. Then, based on the development results of DR cavity No. 0, we fabricated two production versions of the DR cavity, the first (DR cavity No. 1) in fiscal year 2012 and the second (DR cavity No. 2) in fiscal year 2013. Details on the DR cavities are given in [3-8].

Whereas DR cavity No. 2 was used in our previous study [3], DR cavity No. 0 was used in this study. Figure 1(b) shows a photograph of DR cavity No. 0 immediately after its delivery (without any cooling pipes), and typical design parameters for this cavity are summarized in Table I. DR cavities No. 0, No. 1, and No. 2 are based on the same electrical design, have almost the same mechanical structure, and were fabricated using the same method except for the surface protection process after the final machining of the cavities; the end plates of DR cavities No. 1 and No. 2 were electropolished, whereas those of DR cavity No. 0 were chemically etched. Consequently, DR cavity No. 0 has a lower unloaded quality factor $Q_{0}$ of the accelerating mode; $Q_{0}=93 \%$ IACS for DR cavity No. 0 , and $Q_{0}=$ 97\% IACS for DR cavities No. 1 and No. 2, where IACS is the International Annealed Copper Standard. In addition, during high-power operation, DR cavity No. 0 had a radiation level near the cavity approximately five times those of DR cavities No. 1 and No. 2 because of its higher field emission. However, DR cavity No. 0 was successfully conditioned up to an accelerating voltage per cavity (cavity

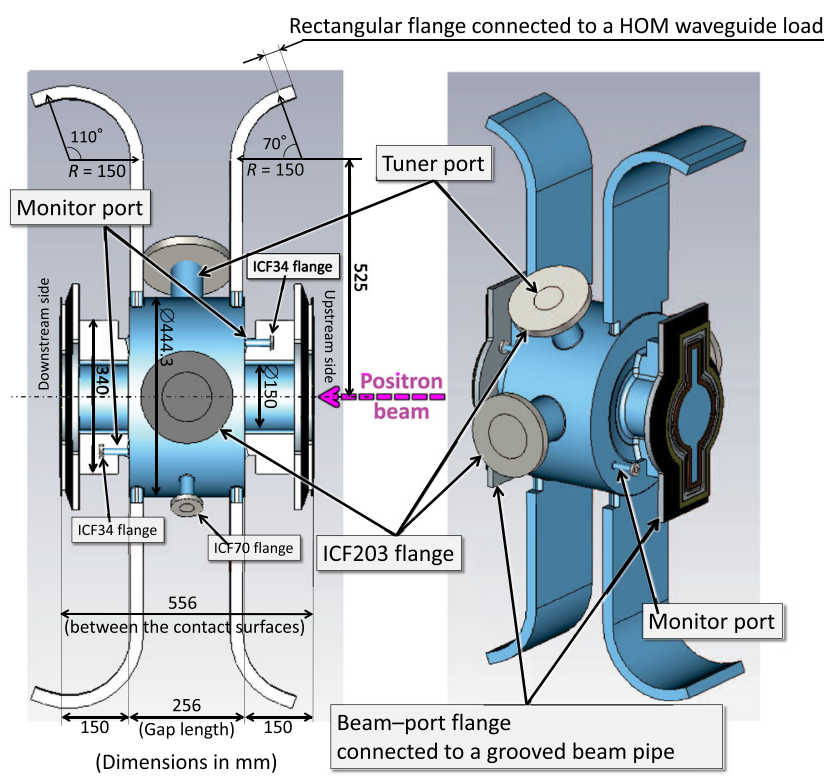

(a) Conceptual diagram of the DR cavity. The blue region is a vacuum during high-power operation. The gap length and diameter of this cavity are 256 and $444.3 \mathrm{~mm}$, respectively.

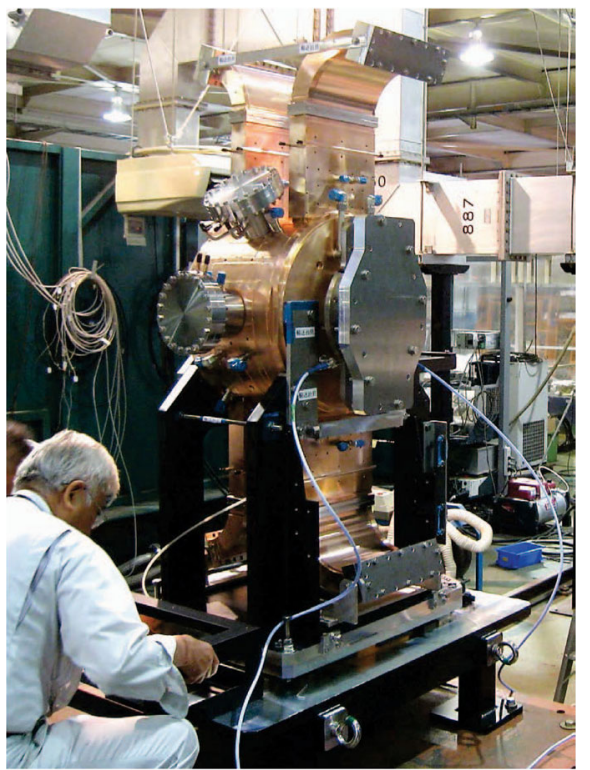

(b) DR cavity No. 0 immediately after its delivery.

FIG. 1. DR cavity.

voltage $V_{c}$ ) of $0.90 \mathrm{MV}$ and an equivalent accelerating gradient $E_{\text {acc }}$ of $3.5 \mathrm{MV} / \mathrm{m}$, which is the radiation limit of the test stand, and it showed a high-power performance comparable to those of DR cavities No. 1 and No. 2. Figure 2 shows the conditioning histories of the three DR cavities up to $V_{c}=0.90 \mathrm{MV}$. DR cavity No. 0 was first high-power tested in 2012, as shown in Fig. 2(a), and then retested in 2017, as shown in Fig. 2(d). In the second highpower test of DR cavity No. 0 in 2017, the rf power increased faster and the vacuum pressure was significantly lower than in its first high-power test in 2012 as a result of 
TABLE I. Design parameters of DR cavity No. 0.

\begin{tabular}{|c|c|}
\hline rf operating frequency & $508.9 \mathrm{MHz}$ \\
\hline$R_{\mathrm{sh}}{ }^{\mathrm{a}} / Q_{0}$ & $150 \Omega$ \\
\hline$Q_{0}$ & $\approx 29000(93 \%$ IACS $)$ \\
\hline Input coupling factor & $\approx 1.3$ \\
\hline Cavity voltage during operation & $\begin{array}{l}V_{c}=0.70 \mathrm{MV} \\
\left(E_{\mathrm{acc}}=2.7 \mathrm{MV} / \mathrm{m}\right)\end{array}$ \\
\hline Cavity voltage of the specification & $\begin{array}{l}V_{c}=0.80 \mathrm{MV} \\
\left(E_{\mathrm{acc}}=3.1 \mathrm{MV} / \mathrm{m}\right)\end{array}$ \\
\hline Wall loss power at $V_{c}=0.70 \mathrm{MV}$ & $\approx 115 \mathrm{~kW}$ \\
\hline Wall loss power at $V_{c}=0.80 \mathrm{MV}$ & $\approx 146 \mathrm{~kW}$ \\
\hline$E_{s}^{(\max ) \mathrm{b}} / E_{\mathrm{acc}}$ & 3.7 \\
\hline$H_{s}^{(\max ) \mathrm{c}} / E_{\mathrm{acc}}$ & $9.6 \mathrm{kA} \mathrm{m}^{-1}(\mathrm{MV} / \mathrm{m})^{-1}$ \\
\hline
\end{tabular}

the memory effect, and the vacuum pressure level in the second test was comparable to those in the high-power tests of DR cavities No. 1 and No. $2\left(\approx 1 \times 10^{-5} \mathrm{~Pa}\right)$, as shown in Figs. 2(b) and 2(c). In addition, the cavity voltage was successfully maintained at a maximum value of $V_{c}=$ $0.90 \mathrm{MV}$ for six hours after if conditioning in DR cavity No. 0; this level of stability is statistically equivalent to the measured breakdown rates of DR cavities No. 1 and No. 2 at $V_{c}=0.90 \mathrm{MV}$ of $5.0_{2.7}^{+4.8}$ per day and $3.3_{-1.0}^{+1.3}$ per day, respectively, where the errors were calculated according to Poisson statistics for a $1 \sigma(68 \%)$ confidence interval. The present study was performed during the second high-power test of DR cavity No. 0 in 2017.

\section{EXPERIMENTAL SETUP}

Because the experimental setup is the same as that in our previous study [3] except for the cameras, only an overview is presented in this section.

Figure 3 shows the setup of the high-power test stand. High rf power was fed into the cavity through a coaxialtype high-power input coupler with a coupling loop at the end of the coaxial line. On each of the upstream and downstream beam ports [9], a vacuum chamber containing an elliptical mirror (mirror chamber) was mounted to allow the direct in-situ observation of the inside of the cavity through the beam port. Figure 4(a) shows a photograph of the mirror, which was made of aluminum. These mirrors were necessary because the radiation level near the beam axis was high during the high-power operation. Three cameras were positioned as shown in Fig. 5 for multidirectional and wide-field observation, where camera 1 was always a high-speed camera, and either both cameras 2 and 3 were high-speed cameras or one was a hyperspectral camera.

Cavity breakdown was detected when the following two conditions were satisfied: (1) the interlock system was activated by the rf power reflected from the cavity exceeding a specified threshold (typically, a few percent of the $\mathrm{rf}$ power input to the cavity) and (2) the signal obtained through the pickup antenna, shown in Fig. 5, on the cavity (pickup signal) decayed much more rapidly than the filling time of approximately $8 \mu \mathrm{s}$. In cavity breakdown, the decay time constant of the pickup signal was much less than $1 \mu \mathrm{s}$, as determined by plasma evolution to cavity breakdown.

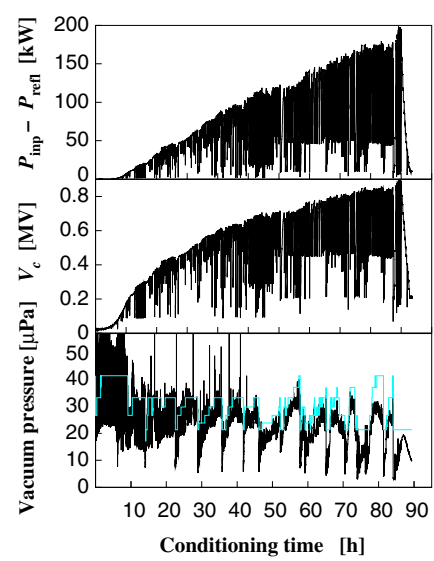

(a)

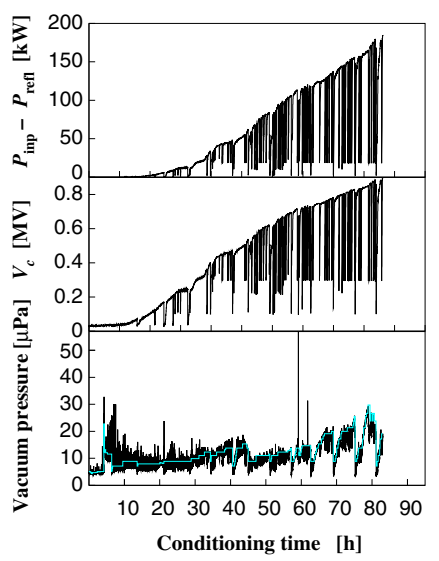

(b)

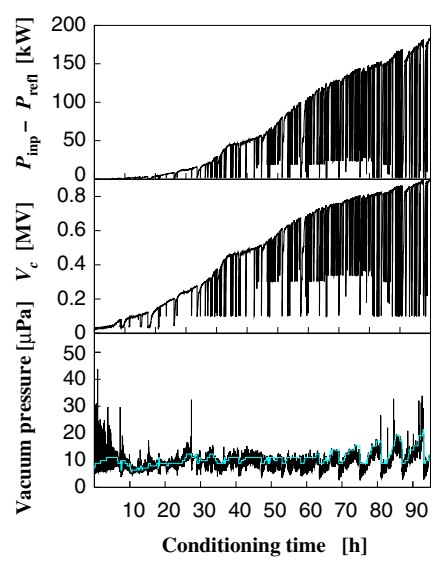

(c)

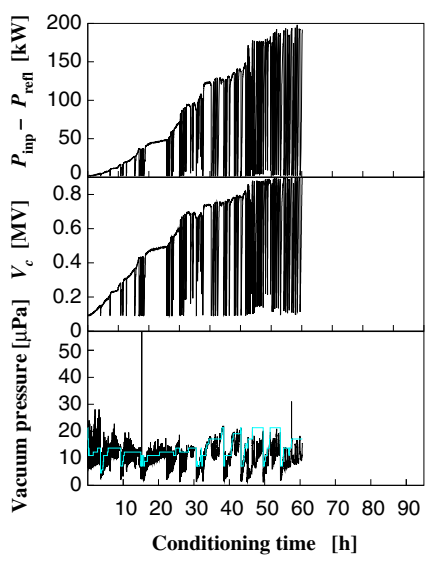

(d)

FIG. 2. Conditioning histories until $V_{c}$ reached $0.90 \mathrm{MV}$ with data points recorded every $10 \mathrm{~s} . P_{\text {inp }}\left(P_{\text {refl }}\right)$ indicates the rf power input to (reflected from) the cavity. Data with no input rf power, data obtained while the input rf power was kept constant, and data used to tune the control system or klystron are excluded here. The light blue lines indicate the reference vacuum pressure specified in the automatic conditioning by computer control. If the vacuum pressure is higher than the reference pressure, the rf power is stepped down until the vacuum pressure becomes lower than the reference pressure, and then the power is gradually stepped up as long as the vacuum pressure remains lower than the reference pressure. The step-up slope is proportional to the difference between the reference and vacuum pressures. (a) DR cavity No. 0 (2012); (b) DR cavity No. 1 (2013); (c) DR cavity No. 2 (2014); (d) DR cavity No. 0 (2017). 


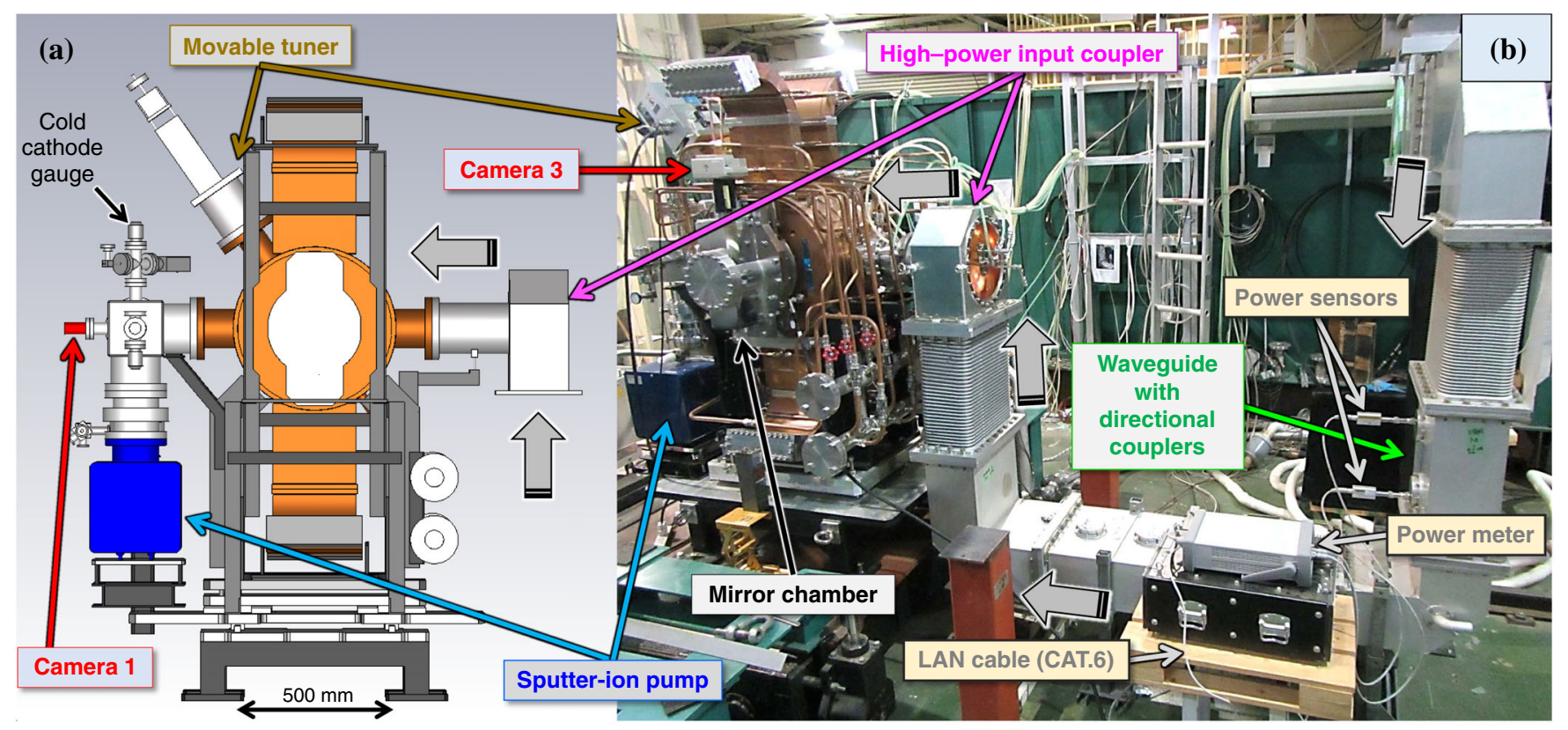

FIG. 3. (a) Diagram of DR cavity No. 0. (b) Photograph of the test stand used for the high-power test of DR cavity No. 0 in a radiationcontrolled area. The bold arrows indicate the flow of high rf power generated by a 1.2-MW continuous-wave klystron. The klystron is not included in this photograph. The turbo-molecular pump is behind the sputter-ion pump. The cavity was cooled by water with a temperature of $30^{\circ} \mathrm{C}$ and a total flow rate of $140 \mathrm{~L} / \mathrm{min}$. No beam was injected into any cavity in this test stand.

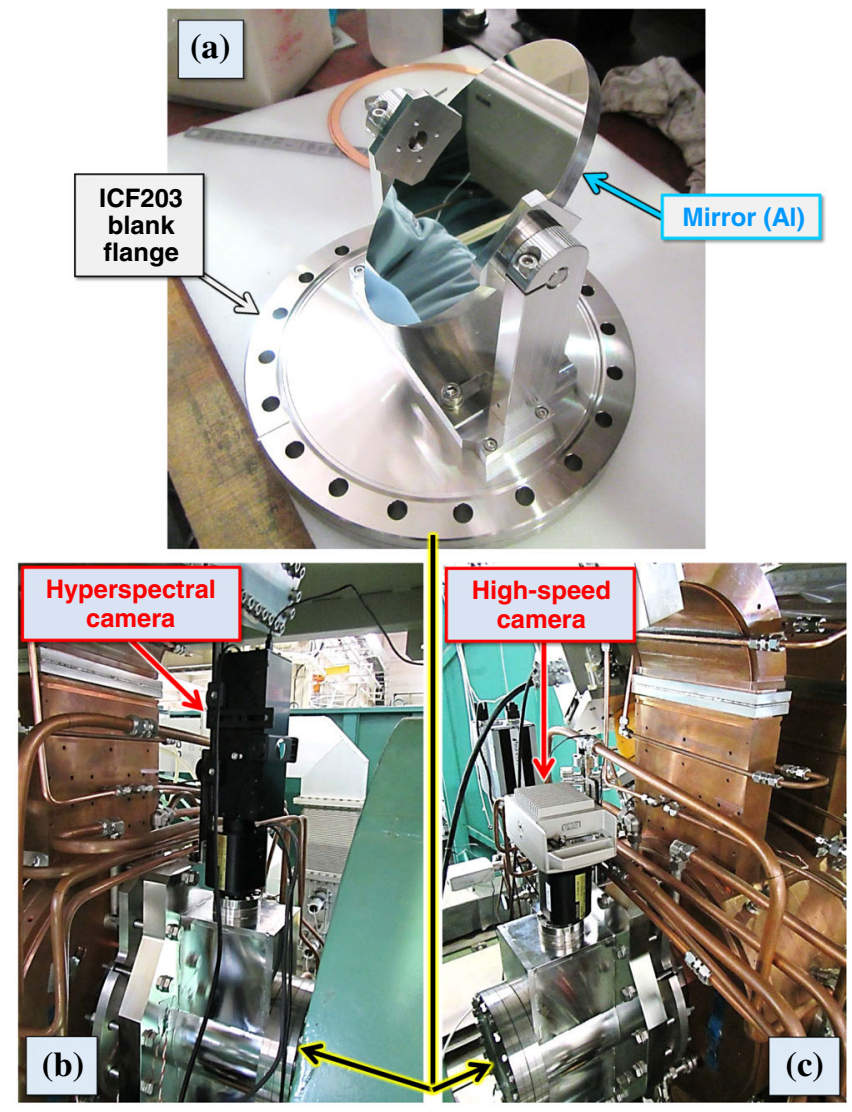

FIG. 4. (a) Elliptical mirror made of aluminum. Setup of the mirror chamber with (b) a hyperspectral camera or (c) a highspeed camera mounted on the view port of the mirror chamber.
Figure 6 shows the surface field of the accelerating mode at $V_{c}=0.90 \mathrm{MV}$; it should be noted here that the upstream and downstream end plates were referred to as the fixed and tuning end plates, respectively, in our previous paper [3]. The maximum surface electric field was $13 \mathrm{MV} / \mathrm{m}$, which is much lower than that required for field evaporation (several gigavolts per meter); however, cavity breakdowns occurred during high-power operation.

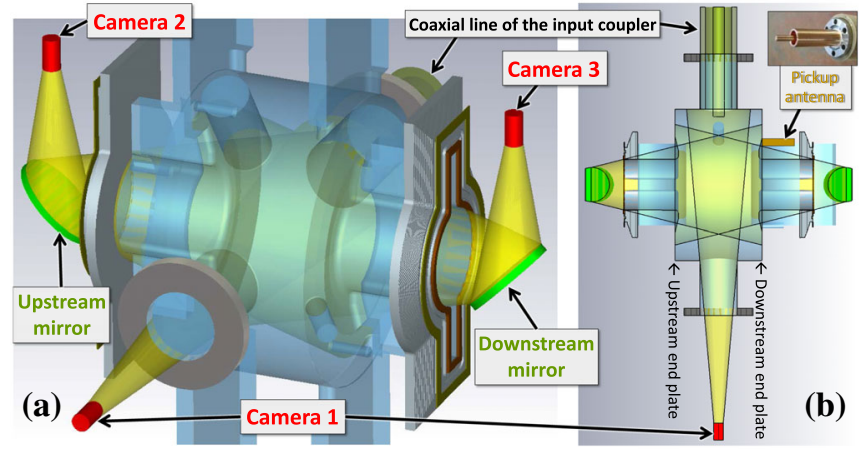

FIG. 5. (a) Schematic of the experimental setup for multidirectional and wide-field observation. The red cylinders and yellow regions represent the three cameras and their fields of view, respectively. During the high-power test, the inside of the cavity (blue) was a vacuum, and the mirrors (green) [shown in Fig. 4(a)] were contained in the mirror chambers. (b) Crosssectional view of the experimental setup along the horizontal plane including the center of the cavity. 

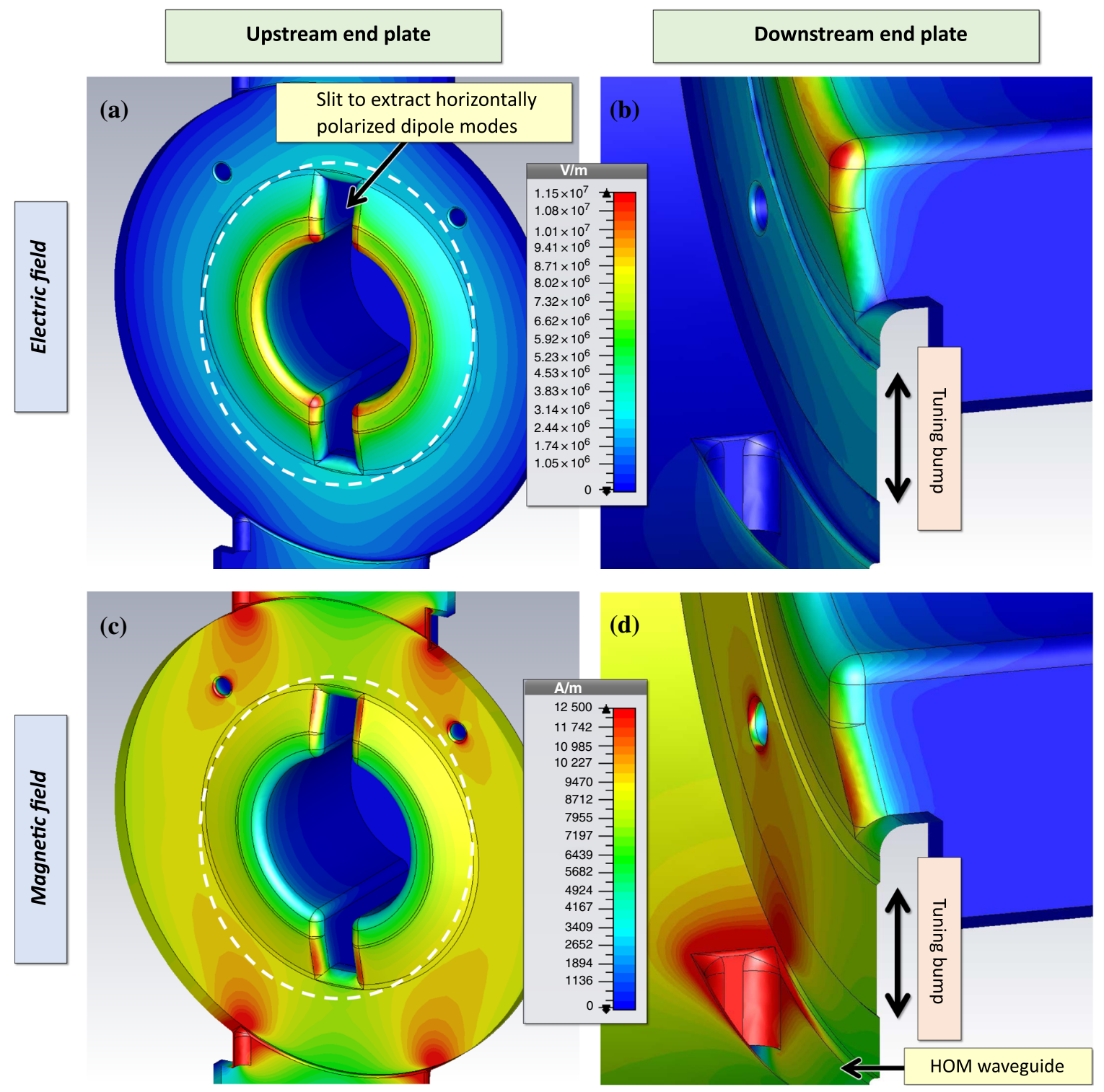

FIG. 6. Surface field of the accelerating mode at $V_{c}=0.90 \mathrm{MV}\left(E_{\mathrm{acc}}=3.5 \mathrm{MV} / \mathrm{m}\right)$, where the gravitational force points vertically downward. The region inside the white dashed ellipse on the upstream end plate was observable with camera 3 , and the observable region on the downstream end plate was almost the same. The maximum strengths of the electric and magnetic fields were $13 \mathrm{MV} / \mathrm{m}$ on the nose cone and $34 \mathrm{kA} / \mathrm{m}$ at the aperture for the HOM waveguide, respectively. The maximum values in the scales for the electric and magnetic fields do not correspond to the maximum field strengths but were selected for visual effect. (a) Electric field distribution on the upstream end plate. (b) Electric field distribution on the downstream end plate with a vertical cut plane. (c) Magnetic field distribution on the upstream end plate. (d) Magnetic field distribution on the downstream end plate with a vertical cut plane.

\section{SPECTRA OF STABLE BRIGHT SPOTS}

\section{A. Hyperspectral camera}

To investigate the light emitters of stable bright spots in Breakdown Type I, their spectra were measured using a hyperspectral camera (NH-KE3, EBA JAPAN Co., Ltd. [10]) as shown in Fig. 4(b). The specifications of the camera are summarized in Table II. This camera is based on line scanning for one-dimensional spectroscopy, and moving the line-scanning system mechanically enables two-dimensional (2D) spectroscopy, as shown in Fig. 7. This 2D spectroscopy enables the spectra of different bright spots on the same end plate to be measured separately and simultaneously. In addition, 2D spectroscopy is necessary to measure the spectra of point light sources like bright spots because the light from a point light source is defocused by dispersion originating in the focus lens of the camera and is detected in a number of pixels of the image sensor. In this case, the spectra observed at different pixels have different shapes for the same point light source; 
TABLE II. Specifications of the hyperspectral camera NH-KE3 used in this study.

\begin{tabular}{ll}
\hline \hline Image sensor & CMOS $^{\mathrm{a}}$ \\
Number of pixels & 360000 \\
Number of bits in digitization & 10 \\
Wavelength range & 400 to $1000 \mathrm{~nm}$ \\
Wavelength resolution & $5 \mathrm{~nm}\left(\mathrm{FWHM}^{\mathrm{b}}\right)$ \\
Enclosure size & Height: $76.0 \mathrm{~mm}$ \\
& Width: $72.4 \mathrm{~mm}$ \\
& Length: $213 \mathrm{~mm}$ \\
Weight & $1030 \mathrm{~g}$ \\
\hline \hline
\end{tabular}

${ }^{\mathrm{a} C M O S}$ : Complimentary metal-oxide-semiconductor.

${ }^{b}$ FWHM: Full width at half maximum.

therefore, it is necessary to integrate the spectra observed at different pixels inside a $2 \mathrm{D}$ region surrounding the defocused and dispersed image detected in the 2D spectroscopy.

\section{B. Pixel sensitivity calibration}

For 2D spectroscopy, the pixel sensitivity of the image sensor must be uniform. To achieve this uniformity, the gain of each pixel was calibrated using a uniform light provided by an integrating sphere. Figure 8 shows the setup of this calibration. Under illumination by the uniform light, the spectra observed in the central region (4\% of the total area) of the image sensor were averaged (black dots in Fig. 9), and the resulting average spectrum was fitted with a piecewise function composed of two fifth-order polynomial functions with different parameters to the left and right of the peak position (solid red curve in Fig. 9). The fitted spectrum was defined as the reference spectrum, and the pixel sensitivity calibration was conducted such that all the spectra observed at different pixels under illumination by the uniform light were obtained as the reference spectra for both the absolute intensity and the wavelength dependence.

The pixel sensitivity calibration was initially performed in 2015 before the delivery of the hyperspectral camera,

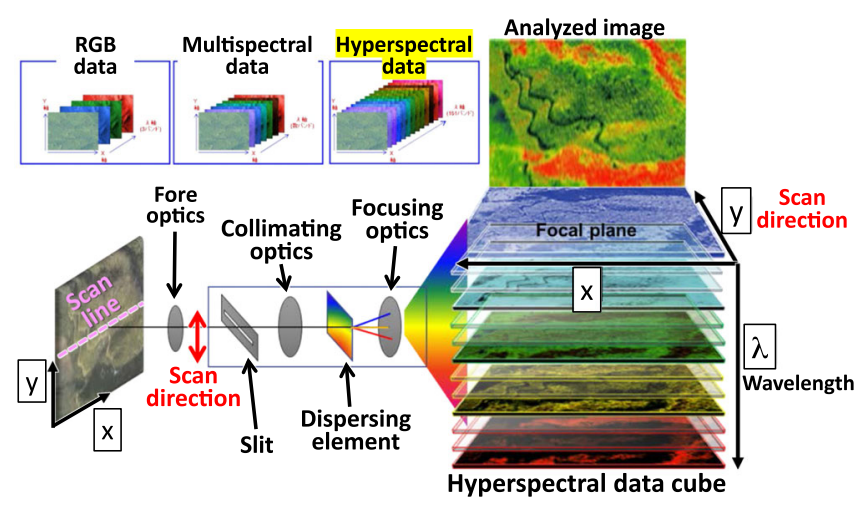

FIG. 7. Schematic of the hyperspectral imaging technology. Courtesy of EBA JAPAN Co., Ltd. [11].

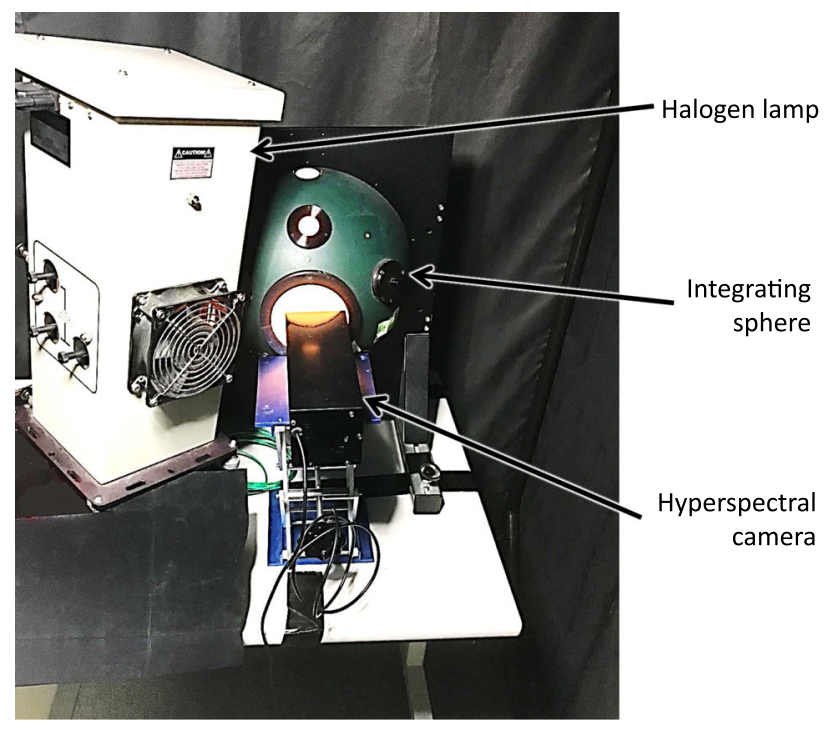

FIG. 8. Setup of the pixel sensitivity calibration using an integrating sphere. Photograph courtesy of EBA JAPAN Co., Ltd.

and a second calibration was performed in 2018 with the same setup as in 2015 after all of the spectrum measurements in this study; the reference spectrum was found to have changed between these two calibrations, as shown in Fig. 9. The reason for this difference has not yet been conclusively determined; however, a possible cause is the occurrence of radiation damage during the high-power test in the test stand. This difference will be included as a

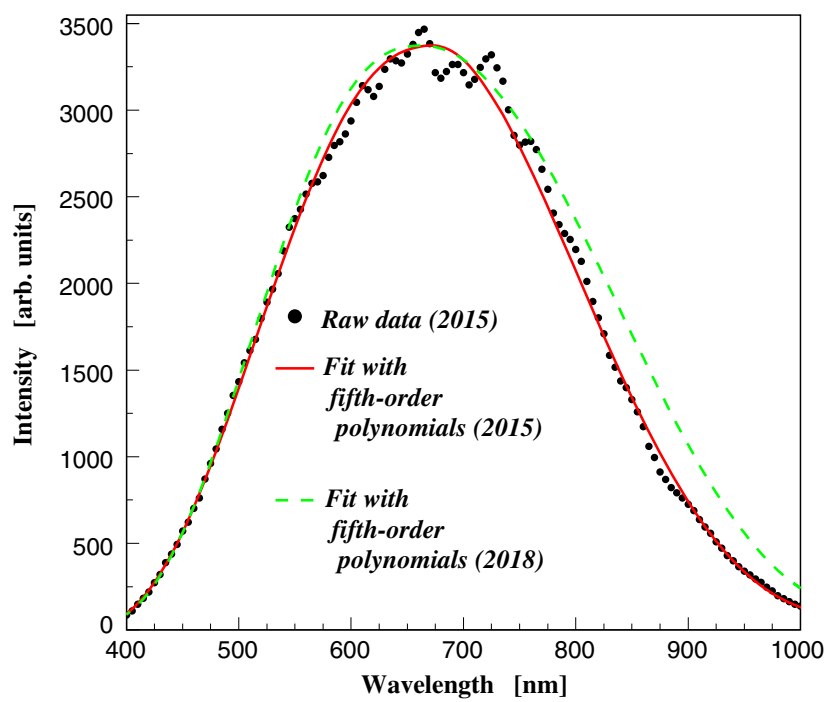

FIG. 9. Spectrum measurements for the uniform light emitted by the integrating sphere. The black dots are raw data taken in 2015 before the delivery of the hyperspectral camera. The solid red curve (dashed green curve) was obtained by fitting the raw data taken in 2015 (2018) with fifth-order polynomials for use as the reference spectrum. The height of the dashed green curve is scaled so that the two reference spectrum curves have the same heights. 
systematic error in the final results presented later in this paper.

\section{Wavelength calibration}

The absolute wavelength was calibrated using a red $\mathrm{HeNe}$ laser, which has a single wavelength of $632.8 \mathrm{~nm}$. As shown in Fig. 10(a), the wavelength resolution of the hyperspectral camera is consistent with the specification given in Table II. On the basis of the wavelength measurements shown in Fig. 10(b), a wavelength calibration of $-5.1 \mathrm{~nm}$ was applied together with the pixel-sensitivity calibration in the following analysis. In addition, before,
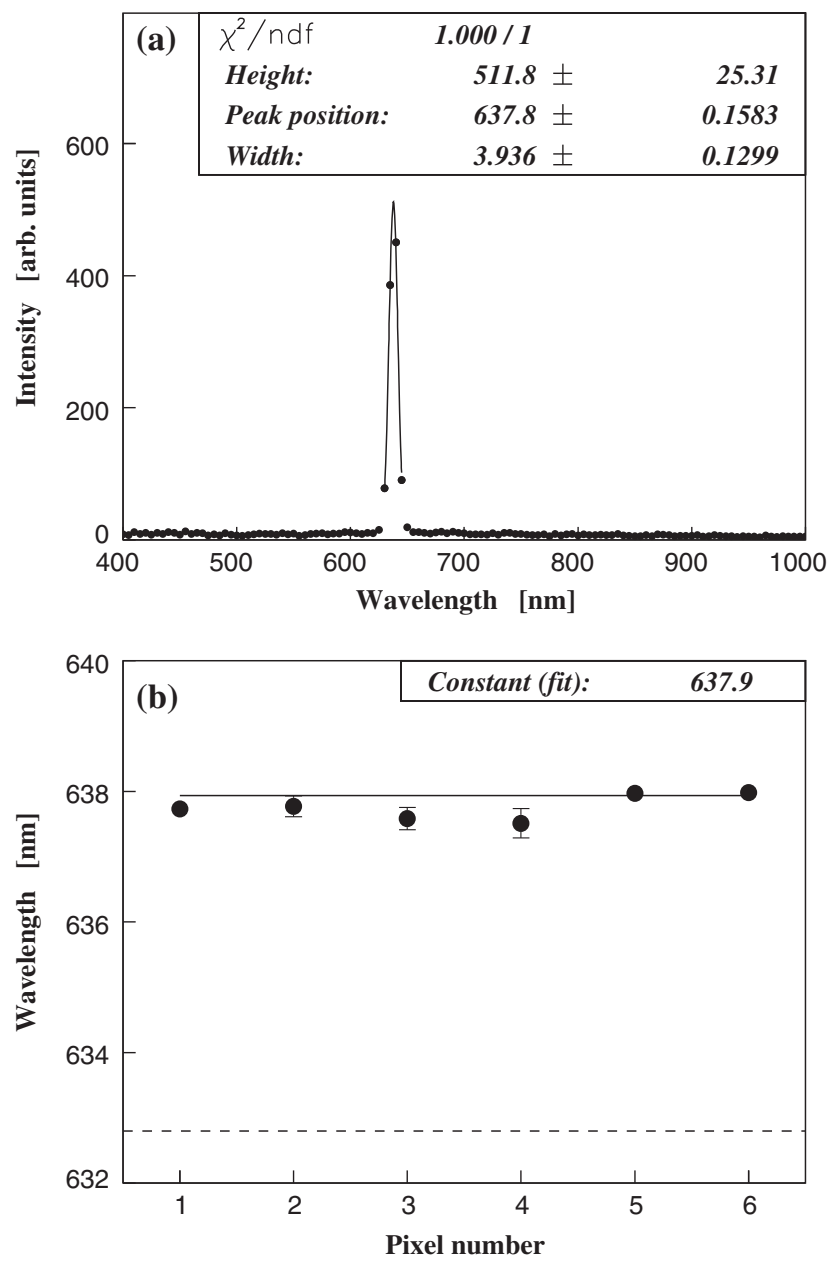

FIG. 10. Observed spectra for the red HeNe laser after applying the pixel sensitivity calibration. (a) Spectrum measured at the central pixel of the image sensor. The black dots represent measurements at intervals of $5 \mathrm{~nm}$, and the solid curve is the result of fitting with a Gaussian function around the peak. The numbers given at the top describe the fitting results, where the errors were calculated such that the ratio of $\chi^{2}$ to the number of degrees of freedom (ndf) is unity. (b) Fitted peak positions at six different pixels on the image sensor. The solid line is the constant fitting result obtained for the six points, and the exact value of this fitting is given at the top part of the plot. The horizontal dashed line indicates the wavelength of the red $\mathrm{HeNe}$ laser $(632.8 \mathrm{~nm})$. during, and after the spectrum measurements conducted in this study, spectra from the same multi-halogen lamp were measured to confirm that the observed wavelengths of the sodium D-lines (589.0 $\mathrm{nm}$ and $589.6 \mathrm{~nm} \mathrm{[12])} \mathrm{did}$ not change.

\section{Correction factor to convert observed spectra to physical spectra}

Even if the pixel sensitivity calibration is applied, the wavelength-dependent efficiency of the hyperspectral camera cannot be corrected; that is, the observed spectra cannot be converted to physical spectra unless the true spectrum of the light source used in the pixel sensitivity calibration, shown in Fig. 8 (halogen lamp), is known. To obtain a correction factor for this conversion, thermal radiation spectra from the high-temperature cylinder made of copper (copper cylinder) shown in Fig. 11(a) were used, and the temperature of the copper cylinder was measured with type-K thermocouples. To heat the copper cylinder, an electron beam of a commercial electron-beam welding (EBW) machine was used. The setup is shown in Fig. 11(b) and 11(c). Six thermocouples were attached to the copper cylinder, and each was fixed with an L-shaped support and
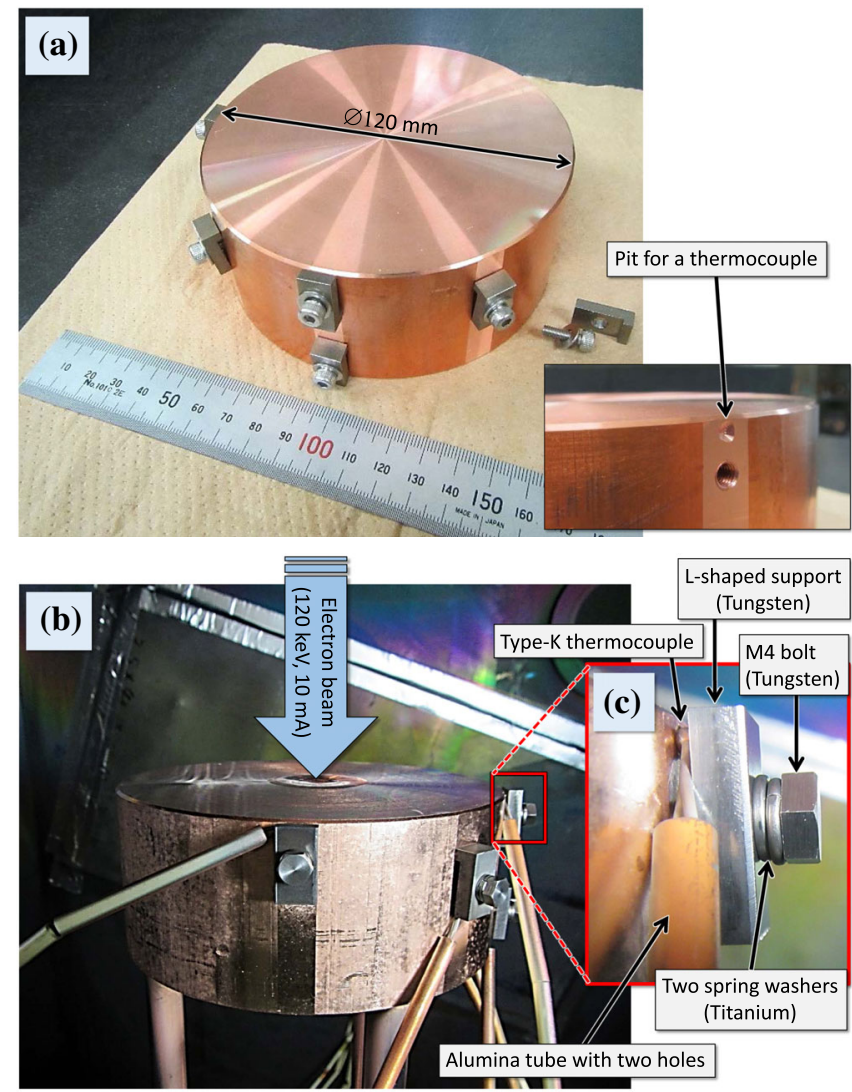

FIG. 11. Setup for the thermal radiation measurement. (a) Copper cylinder with a diameter and a thickness of 120 and $50 \mathrm{~mm}$, respectively. (b) Setup in the EBW machine. (c) Magnified view around one of the thermocouples. 
an M4 bolt, both of which were made of tungsten because of its high melting point.

Thermal radiation spectra from the copper cylinder were measured through a single viewing window in the EBW machine. The viewing window consisted of a pane of lead glass (fixed) and a protective pane of sheet glass (replaceable). The protective sheet glass was replaced before each round of electron beam irradiation. The transmissivity of the viewing window was determined by measuring the spectrum from the same xenon lamp through the window and directly to correct for the effect of the window. Figure 12 shows the measured transmissivity of the viewing window, where the average transmissivity (green curve) was used as a correction for the effect of the viewing window in the following analysis. It should be noted that only the wavelength dependence was considered in this study; throughout this paper, the absolute value of the transmissivity or any spectrum has no physical meaning.

Through electron beam irradiation, the temperature of the copper cylinder was gradually increased to approximately $1000^{\circ} \mathrm{C}$, where the typical vacuum pressure during the irradiation and spectrum measurement was $0.05 \mathrm{~Pa}$. After the peak temperature was reached, the copper was observed to melt at the beam spot, and it was found that the observed spectra changed slightly because of the copper sputtered onto the protective sheet glass of the viewing window. Thus, spectral data obtained before the melting started were used because it was confirmed that there was no such distortion in the observed spectra from that measurement period.

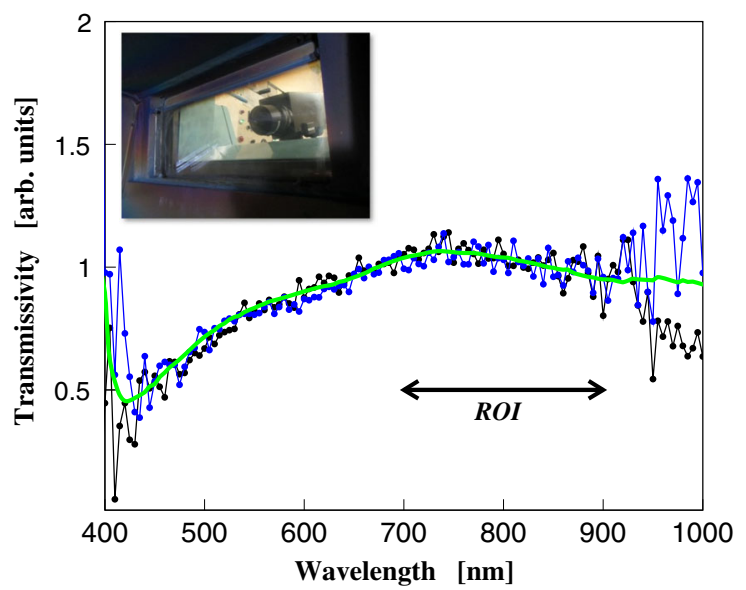

FIG. 12. Transmissivity of the viewing window of the EBW machine. The blue and black points indicate the transmissivity measured at the central pixel of the image sensor and another pixel, respectively. The green curve indicates the transmissivity averaged over all pixels within a central region with an area equal to one quarter of the total area. The top-left inset is a photograph of the hyperspectral camera taken from the inside of the EBW machine through the viewing window. The region labeled ROI indicates the region of interest used to derive physical spectra.
Figure 13(a) shows an image of the copper cylinder taken by the hyperspectral camera at $800 \mathrm{~nm}$ at a temperature of approximately $930^{\circ} \mathrm{C}$, together with the $2 \mathrm{D}$ regions in which spectrum measurements were obtained for the six thermocouples, hereafter referred to as TC1-TC6. It should be emphasized that spectra were measured directly from TC4 and TC5, whereas for the other thermocouples, spectra were measured from the L-shaped supports.

The quality of the data was confirmed by determining the correlation between the temperatures of the thermocouples and the peak positions $\lambda_{p}$ in the observed spectra. Figure 13(b) shows examples of the observed spectra. During the spectrum measurements, no electron beams were generated, and the background spectrum measured
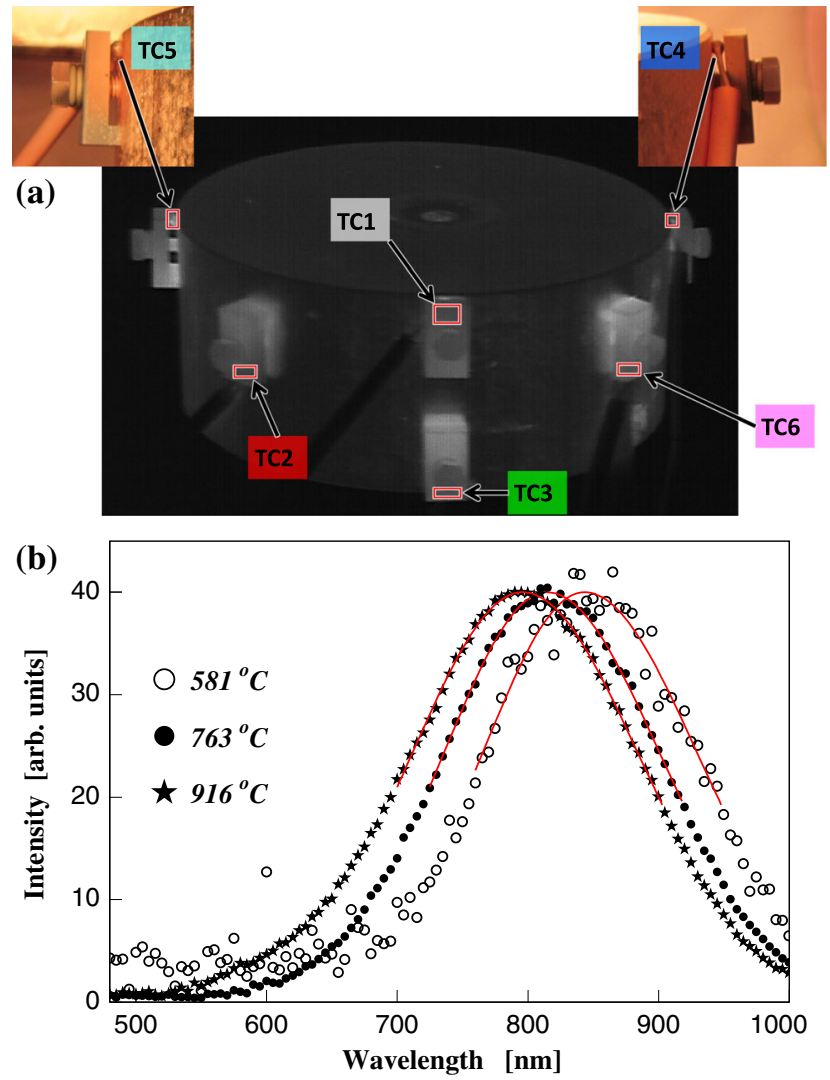

FIG. 13. Measurement of the thermal radiation. (a) Image of the copper cylinder taken by the hyperspectral camera at $800 \mathrm{~nm}$ at a temperature of approximately $930^{\circ} \mathrm{C}$. The red rectangles indicate the $2 \mathrm{D}$ regions in which spectrum measurements were obtained for the six thermocouples (TC1-TC6). (b) Observed thermal radiation spectra from $\mathrm{TC} 4$ at temperatures of $581{ }^{\circ} \mathrm{C}$ (open circles), $763{ }^{\circ} \mathrm{C}$ (closed circles), and $916^{\circ} \mathrm{C}$ (stars) after applying the pixel sensitivity and wavelength calibrations, correction for the transmissivity of the viewing window of the EBW machine, and background subtraction. The red curves show the results of fitting with an asymmetric Gaussian function, where the peak position of the resultant function is equivalent to $\lambda_{p}$. The measured spectra are scaled so that the heights of the resultant functions are equal. 
using the same setup for the copper cylinder at room temperature was subtracted from each measurement result to obtain the final spectra. As demonstrated by these examples, the peak position moved toward lower wavelengths with increasing temperature, as expected. It should be noted that the spectra plotted in Fig. 13(b) do not represent physical spectra, because the wavelengthdependent efficiency of the hyperspectral camera is not corrected. Figure 14(a) shows $\lambda_{p}$ values obtained by fitting curves to the measured spectra for all six thermocouples at different temperatures. This figure clearly shows that the correlations for TC4 and TC5 are very close to each other and separated from the other correlation plots, which is consistent with the $2 \mathrm{D}$ measurement regions shown in Fig. 13(a).

From the spectrum data for TC4 and TC5 at temperatures higher than $600^{\circ} \mathrm{C}$ [13], the correction factor to convert the observed spectra to physical spectra was calculated
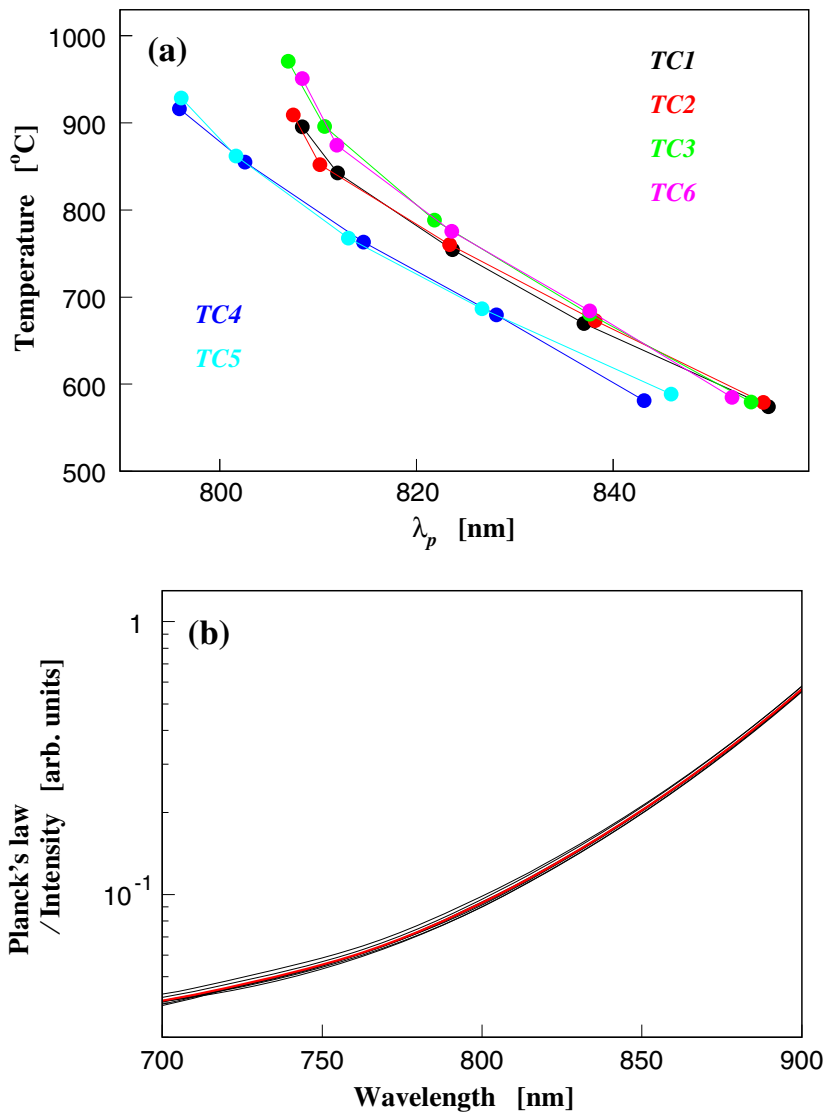

FIG. 14. Results of the thermal radiation measurement. (a) Temperatures of the thermocouples plotted against the peak positions in the thermal radiation spectra. The six thermocouples (TC1TC6) are shown in Fig. 13(a). (b) Correction factor to convert observed spectra to physical spectra. The plot contains eight black curves, which correspond to the data for TC4 and TC5 at the four temperatures above $600{ }^{\circ} \mathrm{C}$ shown in (a). The red curve is the average of the eight black curves. as a function of wavelength by dividing Planck's law (describing physical spectra) by the observed spectra [e.g., Fig. 13(b)]. Planck's law is given by

$$
\mathcal{E} \propto \frac{1}{\lambda^{5}} \frac{1}{e^{\frac{h c}{k k T}}-1},
$$

where $\mathcal{E}$ is the energy density, $\lambda$ is the wavelength, $T$ is the temperature, $c$ is the speed of light, $k$ is the Boltzmann constant, and $h$ is the Planck constant. Figure 14(b) shows the correction factor as a function of the wavelength; in each plot, there are eight black curves corresponding to the four temperature points at each of the two considered thermocouples (TC4 and TC5). The eight black curves are very close to each other, which means that black-body radiation was correctly measured from TC4 and TC5. The average among the eight black curves in Fig. 14(b) (red curve) was used to derive the physical spectra, as described in the following section.

\section{E. Measurement results}

The spectra of the 10 stable bright spots shown in Fig. 15 were measured by integrating the spectra inside a $2 \mathrm{D}$ region surrounding each bright spot, in accordance with the explanation given in Sec. IVA. Correction was applied for the reflectance of the mirror and the transmissivity of the viewing port window of the mirror chamber (mirror chamber correction) in a manner similar to that used to estimate the transmissivity of the viewing window of the EBW machine, although the correction had only a small effect. Figure 16 shows the observed spectra of BS-A1 and BS-A2, which were respectively the brightest and second brightest spots on the upstream end plate, together with that of BS-C1, which was the brightest spot on the downstream end plate. As shown in this figure, there is no line or band spectrum in the visible light or near-infrared regions, and the spectra move toward lower wavelengths at higher cavity voltages. In the same way, no significant line or band spectra were observed for the other bright spots. This observation means that these bright spots are caused by thermal radiation.

The next step was to measure the temperatures of the light emitters of the stable bright spots. Applying the correction factor shown in Fig. 14(b) to the observed spectra yielded the physical spectra; an example of such a physical spectrum is shown in Fig. 17. The spectrum was fitted with Planck's law of Eq. (1), and the temperature was obtained from this fitting, as shown in Fig. 17. The wavelength range was divided into two regions, 700 to $800 \mathrm{~nm}$ and 800 to $900 \mathrm{~nm}$, to address the possibility of a nonuniform temperature distribution. The error of the temperature was calculated such that the $\chi^{2}$ value in the fitting divided by the number of degrees of freedom is unity. Table III shows a list of temperatures obtained using this method for all of the 10 stable bright spots, where the 

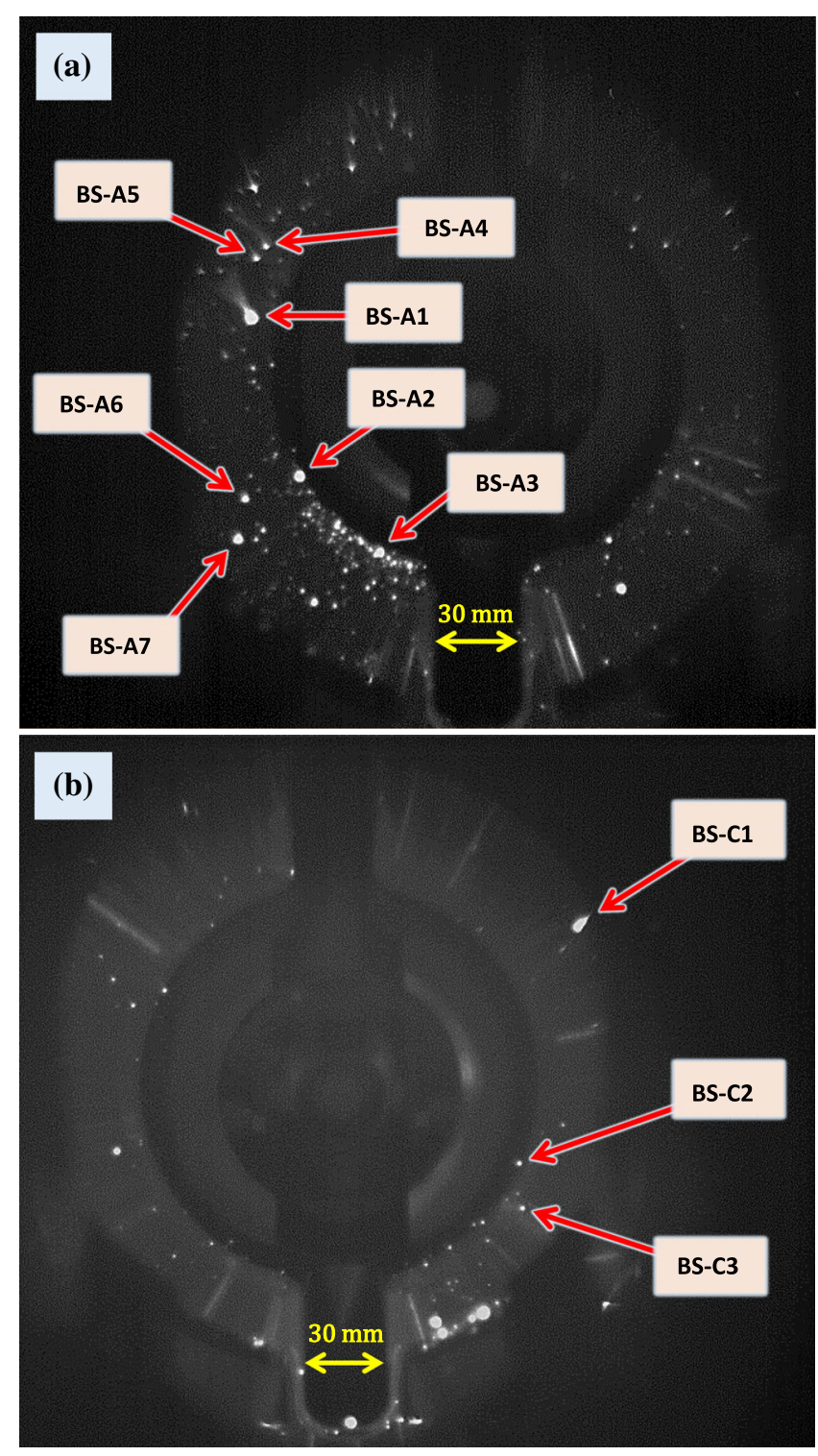

FIG. 15. Snapshots of the (a) upstream and (b) downstream end plates during the high-power test of DR cavity No. 0 at $V_{c}=0.80 \mathrm{MV}$, taken by the high-speed camera. The labels give the names of the bright spots used for reference in this paper.

temperatures were calculated twice based on the two reference spectra shown in Fig. 9 because the reference spectrum in the pixel sensitivity calibration was different. The true value of temperature is expected to be between these two estimates.

\section{SIZE OF THE LIGHT EMITTERS}

The bright spots shown in Fig. 15 have a typical size of $1 \mathrm{~mm}$ on the end-plate surface. However, the light emitters should be smaller. To measure the size of the light emitters, a long-distance microscope (Model K2 DistaMax, Infinity Photo-Optical Company) was used, as shown in Fig. 18(a). A compact TV camera was used in this setup because of the

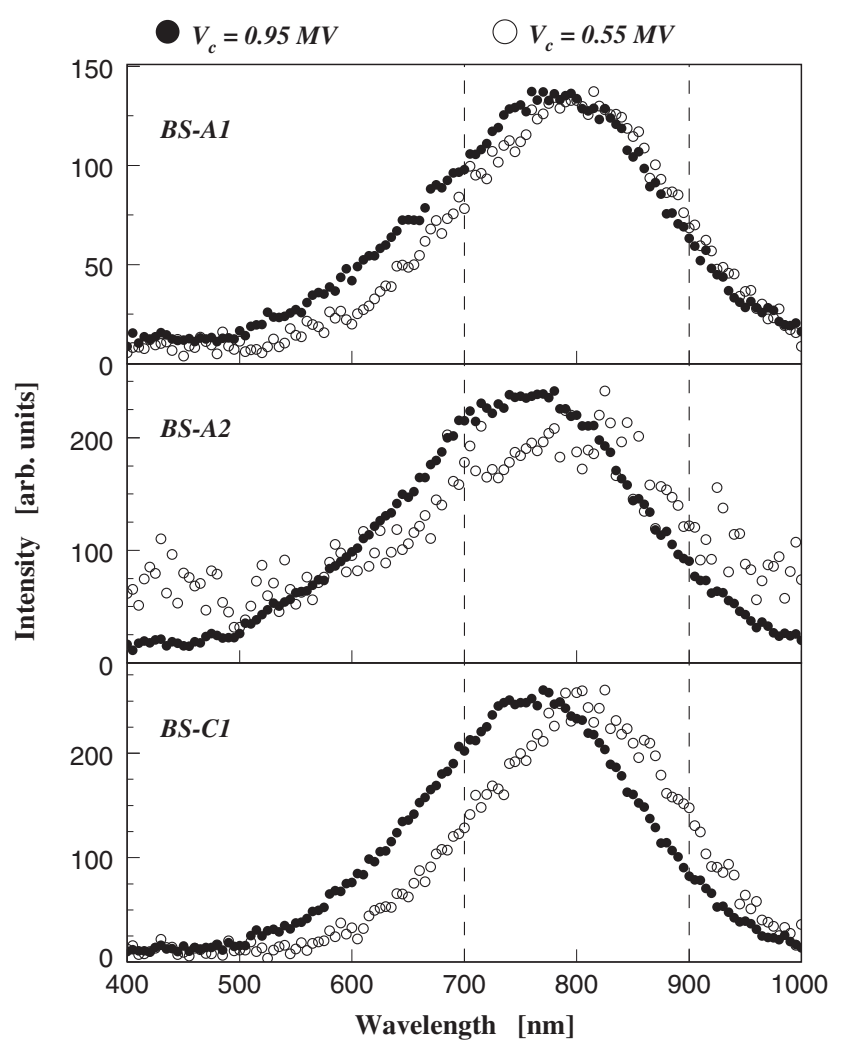

FIG. 16. Observed spectra of the stable bright spots BS-A1, BS-A2, and BS-C1 after applying the pixel sensitivity and wavelength calibrations, the mirror chamber correction, and background subtraction. The vertical dashed lines indicate the region in which the physical spectra were derived.

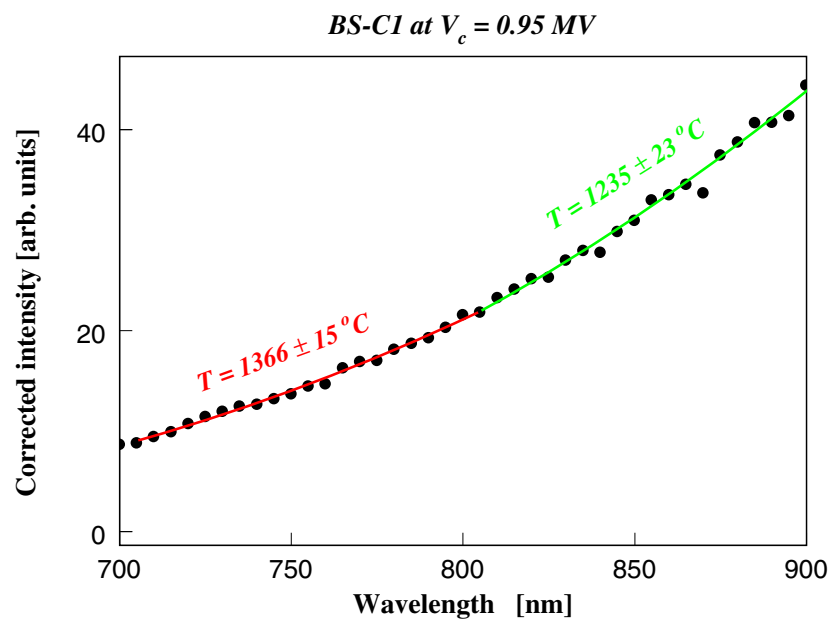

FIG. 17. Example of a physical spectrum (corrected spectrum) obtained for BS-C1. The red and green curves are the results of fitting the data with Planck's law of Eq. (1) in the wavelength regions of 700 to $800 \mathrm{~nm}$ and 800 to $900 \mathrm{~nm}$, respectively. The temperatures obtained from these fitting results are also given. 
TABLE III. Measured temperatures in degrees Celsius of the light emitters of the stable bright spots for the wavelength regions of 700 to $800 \mathrm{~nm}$ and 800 to $900 \mathrm{~nm}$ based on the reference spectrum in the pixel sensitivity calibration performed in 2015 (solid red curve in Fig. 9). Numbers enclosed in square brackets are temperatures calculated on the assumption that the reference spectrum changed to the dashed green curve shown in Fig. 9 before the spectrum measurement of the stable bright spots was initiated. Some stable bright spots were not sufficiently intense to be measured at $V_{c}=0.55 \mathrm{MV}$.

\begin{tabular}{|c|c|c|c|c|}
\hline \multirow[b]{2}{*}{$\begin{array}{l}\text { Name of } \\
\text { bright spot }\end{array}$} & \multicolumn{2}{|c|}{$V_{c}=0.55 \mathrm{MV}$} & \multicolumn{2}{|c|}{$V_{c}=0.95 \mathrm{MV}$} \\
\hline & $\begin{array}{c}700 \text { to } \\
800 \mathrm{~nm}\end{array}$ & $\begin{array}{c}800 \text { to } \\
900 \mathrm{~nm}\end{array}$ & $\begin{array}{c}700 \text { to } \\
800 \mathrm{~nm}\end{array}$ & $\begin{array}{c}800 \text { to } \\
900 \mathrm{~nm}\end{array}$ \\
\hline BS-A1 & $\begin{array}{c}1072 \pm 20 \\
{[1209 \pm 22]}\end{array}$ & $\begin{array}{c}904 \pm 19 \\
{[1124 \pm 27]}\end{array}$ & $\begin{array}{c}1207 \pm 13 \\
{[1369 \pm 14]}\end{array}$ & $\begin{array}{c}1005 \pm 19 \\
{[1266 \pm 26]}\end{array}$ \\
\hline BS-A2 & $\begin{array}{c}1253 \pm 58 \\
{[1428 \pm 72]}\end{array}$ & $\begin{array}{c}865 \pm 68 \\
{[1080 \pm 98]}\end{array}$ & $\begin{array}{c}1440 \pm 20 \\
{[1653 \pm 17]}\end{array}$ & $\begin{array}{c}1178 \pm 31 \\
{[1548 \pm 40]}\end{array}$ \\
\hline BS-A3 & & & $\begin{array}{c}1057 \pm 22 \\
{[1192 \pm 25]}\end{array}$ & $\begin{array}{c}1095 \pm 27 \\
{[1394 \pm 41]}\end{array}$ \\
\hline BS-A4 & & . & $\begin{array}{c}1028 \pm 23 \\
{[1146 \pm 26]}\end{array}$ & $\begin{array}{c}891 \pm 47 \\
{[1109 \pm 66]}\end{array}$ \\
\hline BS-A5 & & & $\begin{array}{c}1316 \pm 38 \\
{[1494 \pm 48]}\end{array}$ & $\begin{array}{c}979 \pm 42 \\
{[1218 \pm 64]}\end{array}$ \\
\hline BS-A6 & & & $\begin{array}{c}1349 \pm 37 \\
{[1560 \pm 46]}\end{array}$ & $\begin{array}{c}859 \pm 34 \\
{[1086 \pm 51]}\end{array}$ \\
\hline BS-A7 & & & $\begin{array}{c}1155 \pm 45 \\
{[1340 \pm 57]}\end{array}$ & $\begin{array}{c}833 \pm 36 \\
{[1052 \pm 53]}\end{array}$ \\
\hline BS-C1 & $\begin{array}{c}933 \pm 26 \\
{[1022 \pm 31]}\end{array}$ & $\begin{array}{c}844 \pm 22 \\
{[1008 \pm 26]}\end{array}$ & $\begin{array}{c}1366 \pm 15 \\
{[1535 \pm 14]}\end{array}$ & $\begin{array}{c}1235 \pm 23 \\
{[1546 \pm 29]}\end{array}$ \\
\hline BS-C2 & & & $\begin{array}{c}1182 \pm 43 \\
{[1325 \pm 49]}\end{array}$ & $\begin{array}{c}1158 \pm 65 \\
{[1502 \pm 95]}\end{array}$ \\
\hline BS-C3 & & & $\begin{array}{c}1250 \pm 33 \\
{[1418 \pm 44]}\end{array}$ & $\begin{array}{c}1088 \pm 71 \\
{[1396 \pm 101]}\end{array}$ \\
\hline
\end{tabular}

limited space. Figure 18(b) shows an image obtained with this setup during the high-power test together with the estimated scale. The size of the spot is approximately $0.1 \mathrm{~mm}$, and no structure can be seen; therefore, the size of the light emitter of BS-C1 is at most $0.1 \mathrm{~mm}$. Other stable bright spots could not be observed because of the limited space and the difficulty in finding such small objects with a long-distance microscope.

\section{OBSERVATION OF FLYING BRIGHT OBJECTS}

To investigate the dynamics of spot-type explosions in Breakdown Type II, direct in-situ observation was performed using three high-speed cameras (HAS-D3M, DITECT Co., Ltd. [14]), as shown in Fig. 4(c); these cameras can be synchronized frame by frame. The maximum frame rate is 100000 frames per second (fps); however, frame rates of only 1000 or $2000 \mathrm{fps}$ were used in this study because the intensity in each frame was too low at frame rates above $2000 \mathrm{fps}$. The specifications of the high-speed camera are summarized in Table IV.

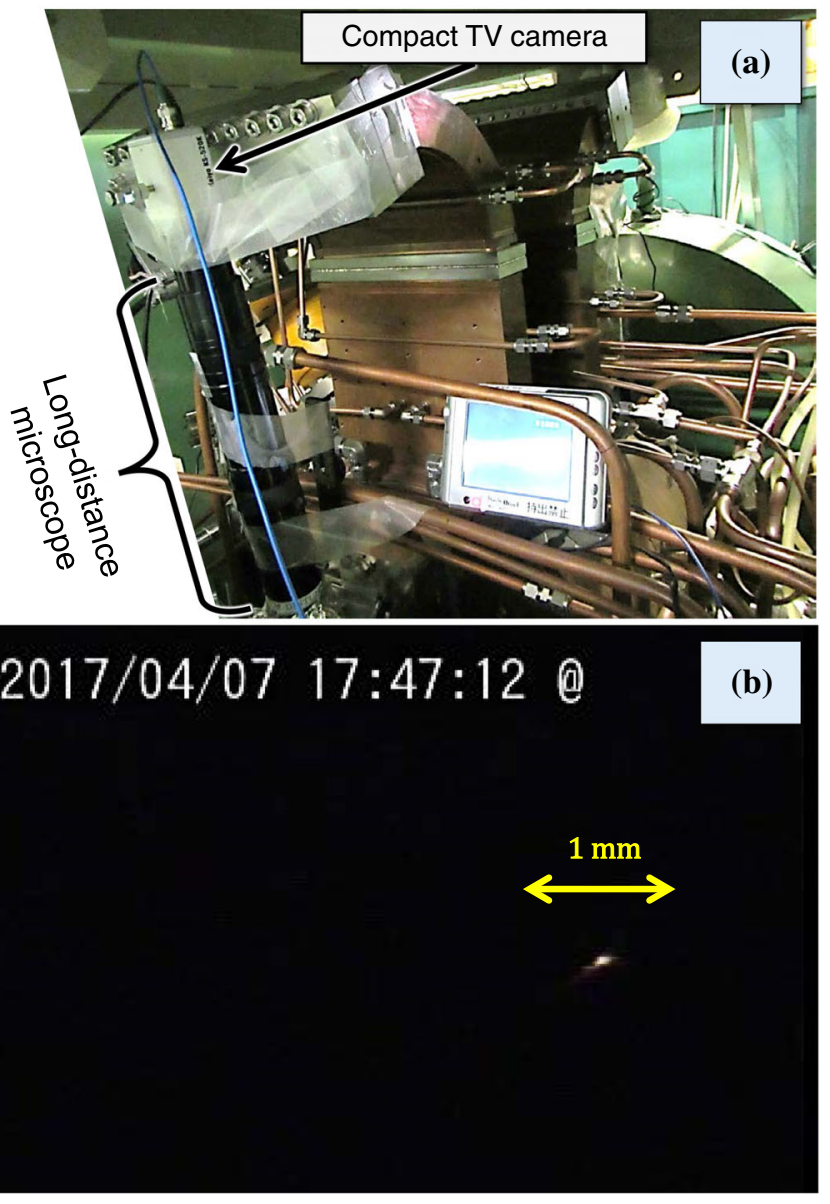

FIG. 18. Magnified image of the stable bright spot BS-C1. (a) Setup with the long-distance microscope. (b) Image obtained at $V_{c}=0.45 \mathrm{MV}$ with the contrast of the image enhanced by $50 \%$ relative to the original contrast.

Videos obtained using the high-speed cameras were automatically saved to a computer storage device during the time period from a few seconds before the interlock system was activated until approximately $10 \mathrm{~ms}$ after this activation. Videos with different trigger timings were stored in different electronic files, each of which typically had a file size of $6 \mathrm{~GB}$ in AVI format.

With the setup using the three high-speed cameras as cameras 1, 2, and 3 in Fig. 5, 40 breakdown events were detected. In four of these events, a flying bright object was

TABLE IV. Specifications of the high-speed camera HAS-D3M used in this study.

\begin{tabular}{ll}
\hline \hline Image sensor & CMOS \\
Number of pixels & 2900000 \\
Frame rate & 100 to $100000 \mathrm{fps}$ \\
Enclosure size & Height: $104 \mathrm{~mm}$ \\
& Width: $160 \mathrm{~mm}$ \\
Weight & Length: $82 \mathrm{~mm}$ \\
\hline \hline
\end{tabular}




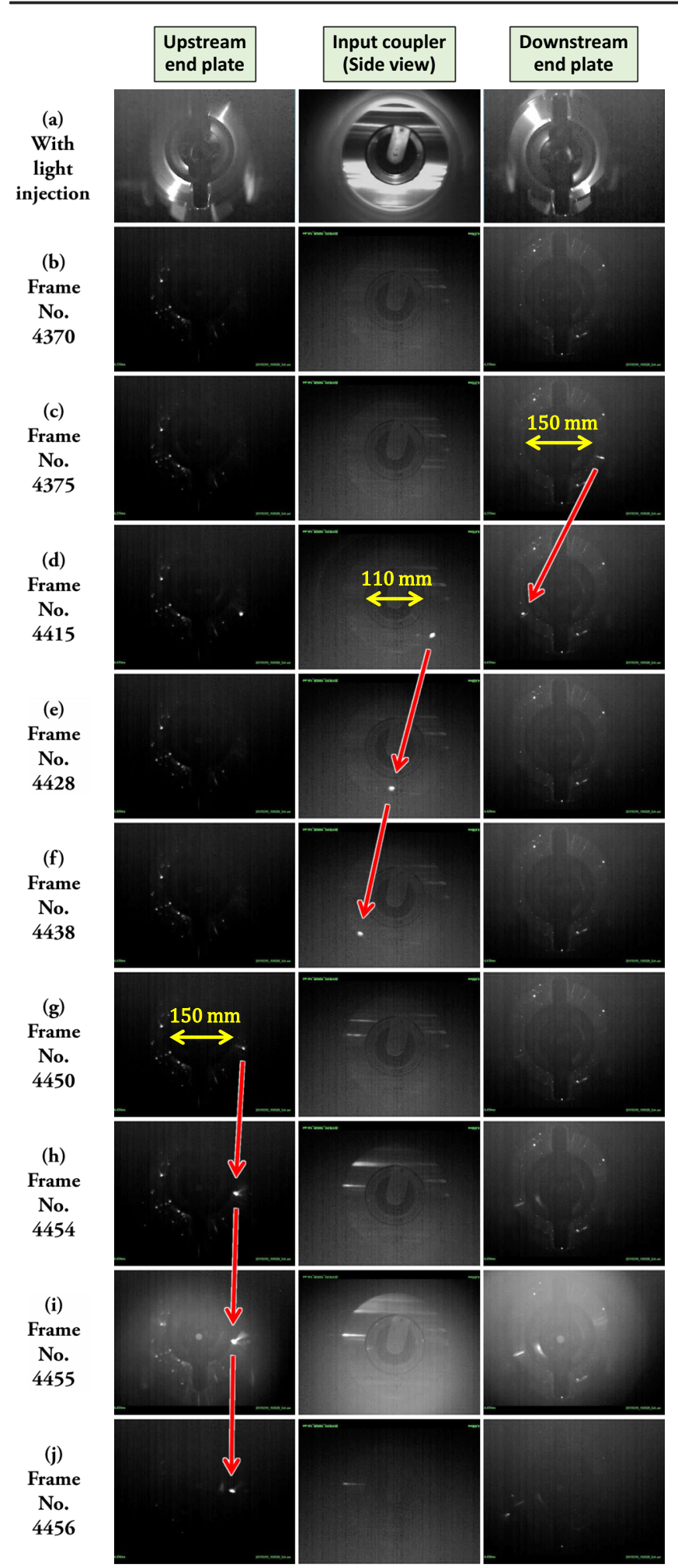

FIG. 19. First breakdown event accompanied by a flying bright object, which occurred at $V_{c}=0.88 \mathrm{MV}, 15: 05$ on March 15 in 2017. (a) Photographs showing the view of the high-speed cameras with light injected into the cavity for visualization. (b)-(j) Snapshots synchronized among the three high-speed cameras recorded at 1000 fps. Red arrows indicate the flying bright object of interest. The brightness of all images was enhanced by $60 \%$ relative to the original brightness. observed to impact the end-plate surface at the moment of cavity breakdown. Figure 19 shows one of these four events, where the gravitational force is directed vertically downward in each snapshot [15]. Nothing abnormal was observed until frame No. 4370 [Fig. 19(b)]. A bright spot appeared at 4 o'clock on the downstream end plate after frame No. 4370; it then moved toward the upstream end plate and impacted the surface of the end plate in frame No. 4455 [Fig. 19(i)]. In the next frame (No. 4456), the intensities of the stable bright spots dropped; therefore, cavity breakdown occurred at the moment of impact observed in frame No. 4455, which means that the impact was a breakdown trigger. In three other breakdown events of the same type, a flying bright object impacted the surface of the downstream end plate at the moment of cavity breakdown, as shown in Fig. 20. The event shown in Fig. 20(c) can be understood as a flying object perpendicularly impacting the end-plate surface and breaking into three pieces.

\section{DISCUSSION}

\section{A. Breakdown type I}

It was found that the light emitters of stable bright spots could have temperatures exceeding $1000^{\circ} \mathrm{C}$. Crucially, the bright spots observed in this study were stable for hours under exposure to ultrahigh vacuum. The vacuum pressure measured by a cold cathode gauge (gauge pressure) during the high-power test was typically $1 \times 10^{-5} \mathrm{~Pa}$, as shown in Fig. 2(d). The actual vacuum pressure inside the cavity was estimated using a Monte Carlo simulator Molflow+ [16] and found to be approximately $2 \times 10^{-5} \mathrm{~Pa}$ at a gauge pressure of $1 \times 10^{-5} \mathrm{~Pa}$. Because the pressure at the triple point of copper is approximately $0.05 \mathrm{~Pa}$, which is obtained using two independent calculators [17,18], any copper material exposed to ultrahigh vacuum sublimates if its temperature is higher than the sublimation point. Figure 21 shows the sublimation curve of copper at pressures below the triple point. The temperature of any copper solid material exposed to ultrahigh vacuum cannot exceed $1000^{\circ} \mathrm{C}$, which means that the light emitters of the stable bright spots are not copper. Candidates of the material should have a high melting point, which is true of carbon, molybdenum, tantalum, tungsten, etc. Therefore, Breakdown Type I can be understood as a physical process in which a microparticle composed of such materials at a high temperature exceeding $1000{ }^{\circ} \mathrm{C}$ interacts with other materials, leading to an explosion, which ignites a vacuum arc and is followed by cavity breakdown; however, neither the mechanism of the explosion nor mechanism disrupting the stability of stable bright spots is currently understood. In addition, the mechanism of heat generation, which is expected to be related to surface currents and/or field emissions, that produces such high temperatures is also not understood and remains as a target of investigation for future studies. 

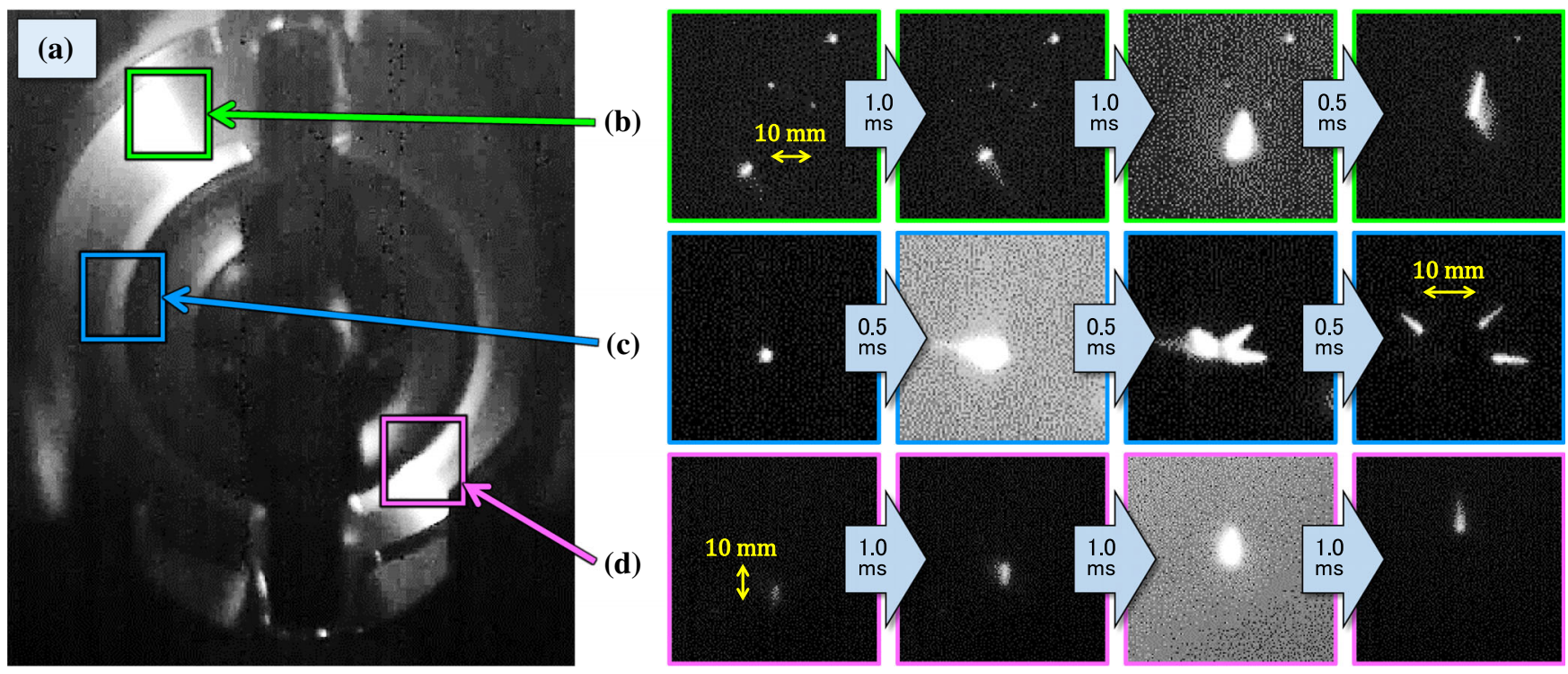

FIG. 20. Three other breakdown events accompanied by a flying bright object. The brightness and contrast of all of the images were enhanced by $60 \%$ and $40 \%$ relative to the original brightness and contrast, respectively. (a) Photograph showing the view of the highspeed camera directed toward the downstream end plate with light injected into the cavity for visualization. Snapshots of the (b) second, (c) third, and (d) fourth breakdown events around the time of cavity breakdown. These are magnified views of the regions shown with (b) green, (c) blue, and (d) magenta squares in (a). These breakdown events occurred in 2017 at (b) $V_{c}=0.89$ MV, $12: 14$ on March 21, (c) $V_{c}=0.89 \mathrm{MV}, 14: 29$ on March 17, and (d) $V_{c}=0.79 \mathrm{MV}, 15: 46$ on March 13.

\section{B. Breakdown type II}

In our previous study [3], in which less-advanced TV cameras with a frame rate of approximately $30 \mathrm{fps}$ were used, one breakdown event that could be interpreted to have been accompanied by a flying bright object impacting the end-plate surface at the moment of cavity breakdown was observed out of a total of 205 detected breakdown events; however, the observed flying object was not very clear because the frame rate was low and the three TV cameras

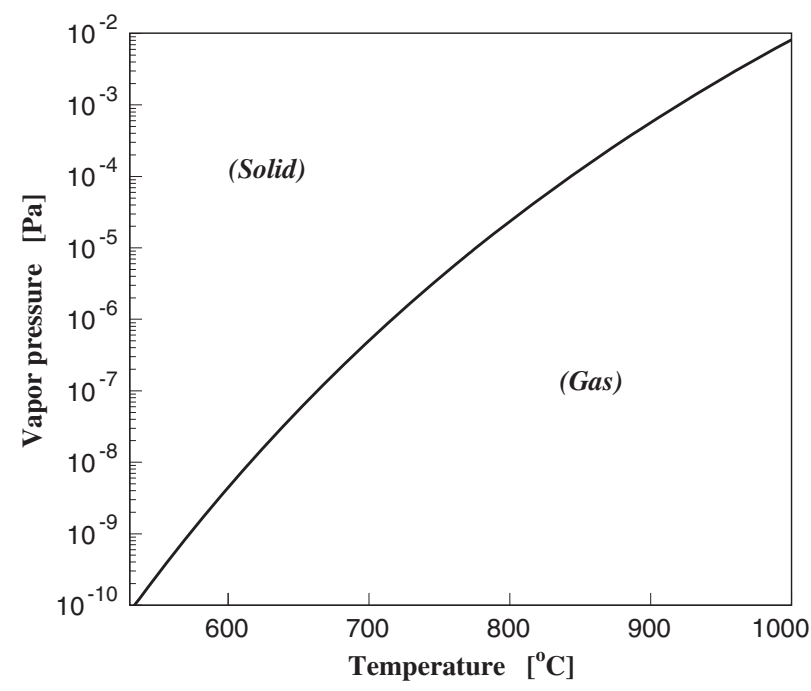

FIG. 21. Sublimation curve of copper. Data from [17], which is consistent with the data obtained using THERMO-CALC [18]. were not synchronized. In the present study, four such events were observed out of 40 total breakdown events detected using the high-speed cameras. It is expected that more such events could be observed using cameras with even higher specifications, namely higher speed and higher sensitivity, particularly in the infrared region. If this is true, Breakdown Type II can be understood as a physical process in which a charged microparticle accelerates and impacts an end-plate surface, leading to an explosion at the surface which ignites a vacuum arc and is followed by cavity breakdown; however, at a low frame rate, such as $30 \mathrm{fps}$, Breakdown Type II might be observed as being accompanied by a spot-type explosion not originating from a stable bright spot. Whether the impact of a flying object on a cavity surface is a major breakdown trigger will be a target of investigation in future studies.

The temperatures of the flying bright objects were not measured; however, they are expected to be high like those of the stable bright spots because despite traveling at a low speed on the order of $10 \mathrm{~m} / \mathrm{s}$, the flying bright objects caused an explosion on the end-plate surface, as shown in Figs. 19 and 20. As shown in Fig. 22, temperatures were estimated using a simple spherical model to represent a flying bright object. Exact electromagnetic-field calculations including skin depth effects were performed for conducting materials of graphite, molybdenum, and copper using CST STUDIO SUITE [19]. Figure 22(c) shows the case when a microparticle at room temperature on the cavity surface, i.e., well cooled by the cavity surface, is detached and heated by currents associated with a $508.9-\mathrm{MHz}$ 

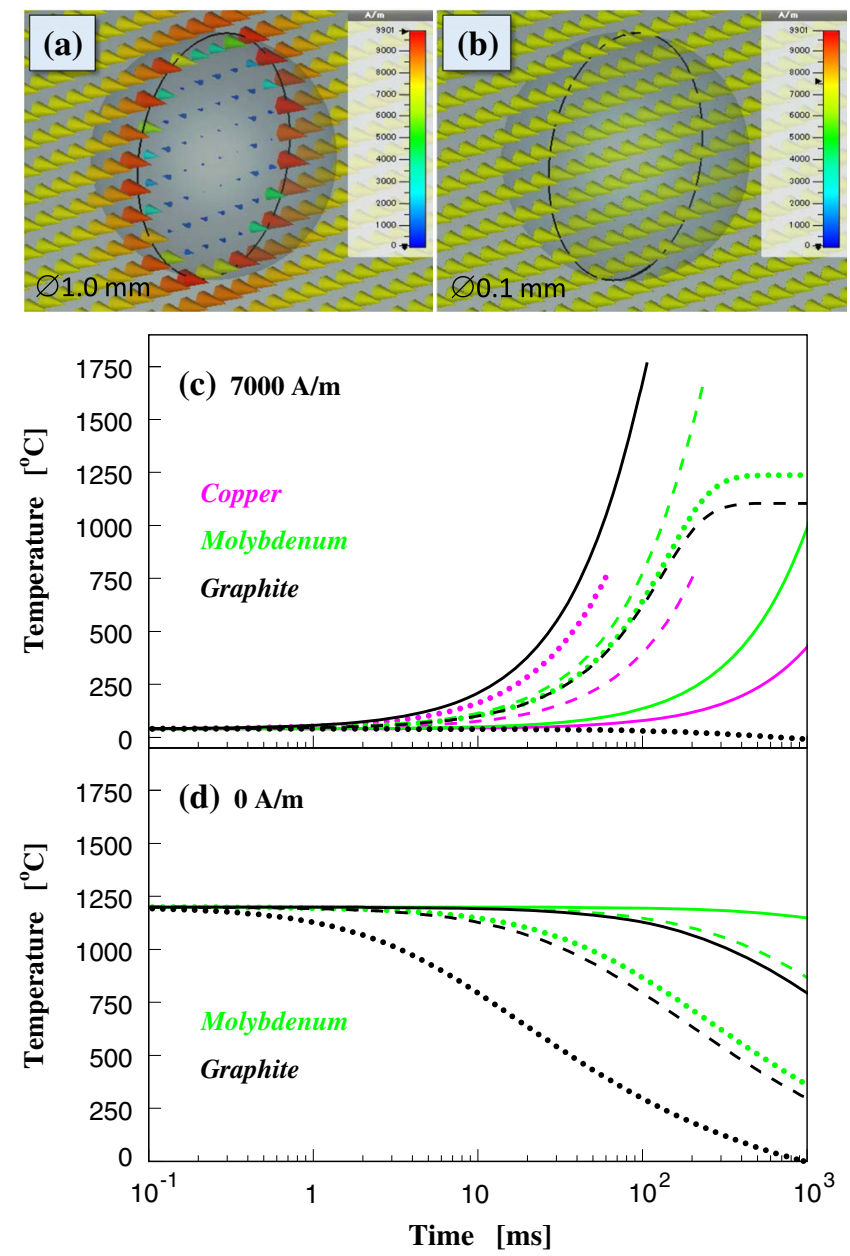

FIG. 22. Simulations of temperatures of spherical microparticles made of graphite (black lines), molybdenum (green lines), and copper (magenta lines) with diameters of $1.0 \mathrm{~mm}$ (solid lines), $0.1 \mathrm{~mm}$ (dashed lines), and $0.01 \mathrm{~mm}$ (dotted lines) located in a vacuum. Radiation cooling was calculated according to the Stefan-Boltzmann law with an emissivity of 0.8 for graphite and 0.1 for copper and molybdenum. Heat capacities of $0.71,0.39$, and $0.28 \mathrm{~kJ} / \mathrm{K} / \mathrm{kg}$ were used for graphite, copper, and molybdenum, respectively. (a) and (b) Application of a 508.9-MHz magnetic field of $7000 \mathrm{~A} / \mathrm{m}$ to graphite microparticles with diameters of 1.0 and $0.1 \mathrm{~mm}$, respectively, assuming an electric conductivity of $1.0 \times 10^{5} \mathrm{~S} / \mathrm{m}$ for graphite. (c) Temperature variation of the microparticles from an initial temperature of $40^{\circ} \mathrm{C}$ with heat generation by a magnetic field of $7000 \mathrm{~A} / \mathrm{m}$. (d) Temperature variation of the microparticles from an initial temperature of $1200^{\circ} \mathrm{C}$ without heat generation.

magnetic field of $7000 \mathrm{~A} / \mathrm{m}$ in a vacuum. Data above the sublimation points at $2 \times 10^{-5} \mathrm{~Pa}\left(1770^{\circ} \mathrm{C}\right.$ for graphite, $1700^{\circ} \mathrm{C}$ for molybdenum, and $800^{\circ} \mathrm{C}$ for copper) are excluded. The selected magnetic field strength of $7000 \mathrm{~A} / \mathrm{m}$ is roughly equivalent to the strength of the field applied to the bright spot BS-A1 at $V_{c}=0.95 \mathrm{MV}$. The electric field was assumed to negligibly contribute to increasing the temperature of the microparticle through field emissions because electric-field enhancement factors are less than three for this model of a conductive sphere with a diameter much less than the wavelength of the electromagnetic field. Additionally, the maximum accelerating gradient is $3.5 \mathrm{MV} / \mathrm{m}$, which means that the surface electric field strength is at most a few tens of megavolts per meter, too low for field emissions to be significant.

It was found that smaller copper microparticles show a more rapid increase in temperature because the skin depth of approximately $0.003 \mathrm{~mm}$ is sufficiently smaller than the microparticles. However, smaller graphite microparticles showed a slower increase in temperature (or a temperature decrease for a size of $0.01 \mathrm{~mm}$ ) because the skin depth is large (approximately $0.07 \mathrm{~mm}$ ) and the magnetic field penetrates microparticles of $0.1 \mathrm{~mm}$ in size, as shown in Fig. 22(b); in this case, the magnetic field is negligibly enhanced around the microparticle. The maximum magnetic field strength around the smaller microparticle was $7100 \mathrm{~A} / \mathrm{m}$ [Fig. 22(b)], whereas that around the larger microparticle was $9900 \mathrm{~A} / \mathrm{m}$ [Fig. 22(a)]. This effect was also seen in the case of the molybdenum microparticle with a size of $0.01 \mathrm{~mm}$ [dotted green line in Fig. 22(c)], where the skin depth is approximately $0.005 \mathrm{~mm}$. This molybdenum microparticle and the graphite microparticle with a size of $0.1 \mathrm{~mm}$ reached an equilibrium state between heat generation and radiation cooling at approximately 1100 to $1200^{\circ} \mathrm{C}$ after a few hundred milliseconds. From the observation of the flying bright object shown in Fig. 19, which had a recorded time of flight of $85 \mathrm{~ms}$, a time of flight of a few hundred milliseconds could be possible.

Figure 22(d) shows the case when a high-temperature microparticle on the cavity surface, like a light emitter of a stable bright spot, is detached and cooled down in a vacuum with no significant field. The molybdenum microparticles (even those of $0.01 \mathrm{~mm}$ in size) and graphite microparticles of at least approximately $0.1 \mathrm{~mm}$ in size can maintain their temperatures up to approximately $10 \mathrm{~ms}$. However, graphite microparticles of approximately $0.01 \mathrm{~mm}$ in size or smaller are rapidly cooled down on a time scale of milliseconds.

Figure 22(c) and 22(d) indicate that graphite microparticles of less than $0.01 \mathrm{~mm}$ in size are not a candidate for "hot" flying objects because they are not significantly heated by the magnetic field and are always rapidly cooled down. On the other hand, graphite microparticles larger than $0.01 \mathrm{~mm}$ in size and molybdenum microparticles are possible candidates. Graphite and molybdenum are simply representative candidates for flying objects; other materials with high melting or sublimation points and an appropriate size could also be considered as candidates.

\section{CONCLUSIONS}

Using the hyperspectral camera, we made the first measurement of the spectra and temperatures of stable bright spots, which are known to be breakdown trigger seeds, and discovered that the light emitters of stable bright spots are not copper but contamination microparticles with 
a high melting or sublimation point (higher than $1000^{\circ} \mathrm{C}$ at a vacuum pressure of $2 \times 10^{-5} \mathrm{~Pa}$ ). In addition, using the high-speed cameras, we made the novel observation of breakdown events accompanied by a flying bright object impacting the end-plate surface, triggering cavity breakdown, which means that such flying bright objects are also breakdown trigger seeds.

On the basis of the observations in this study, we formed the following hypotheses: (1) Breakdown Type I can be understood as a physical process in which a contamination microparticle on a cavity surface with a temperature exceeding $1000{ }^{\circ} \mathrm{C}$ interacts with other materials at the same position, leading to an explosion which ignites a vacuum arc and is followed by cavity breakdown; and (2) Breakdown Type II can be understood as a physical process in which a high-temperature charged microparticle accelerates and impacts the end-plate surface, leading to an explosion on the surface which ignites a vacuum arc and is followed by cavity breakdown.

Some mechanisms related to stable bright spots remain unexplained, specifically regarding the heat generation required to reach the observed high temperatures, the disruption of their stability, and the explosion; these remain as targets of investigation for future studies. In addition, whether high-temperature flying objects are major breakdown trigger seeds for Breakdown Type II remains to be investigated, particularly in the infrared region.

\section{ACKNOWLEDGMENTS}

This work was supported by Grant-in-Aid for Scientific Research (JSPS KAKENHI, Grant No. 15H03671). We are grateful to Hitoshi Inoue, who performed the EBW under the support service of the KEK Mechanical Engineering Center.

\section{APPENDIX: LIST OF ABBREVIATIONS}

The abbreviations used in this paper are listed in Table V.

TABLE V. Abbreviations used in this paper.

\begin{tabular}{ll}
\hline \hline Abbreviation & Full letters \\
\hline BS & Bright spot \\
CMOS & Complimentary metal-oxide-semiconductor \\
DR & Damping ring \\
EBW & Electron-beam welding \\
fps & Frames per second \\
HOM & Higher-order mode \\
FWHM & Full width at half maximum \\
IACS & International annealed copper standard \\
ICF & International conflat flange \\
ndf & Number of degrees of freedom \\
rf & Radio frequency \\
TC & Thermocouple \\
TV & Television \\
2D & Two-dimensional \\
\hline \hline
\end{tabular}

[1] "7th International Workshop on Mechanisms of Vacuum Arcs (MeVArc2018)." https://indico.cern.ch/event/680402/, 2018.

[2] "11th International Workshop on Breakdown Science and High Gradient Technology (HG2018)." https://indico.cern .ch/event/675785/, 2018.

[3] T. Abe, T. Kageyama, H. Sakai, Y. Takeuchi, and K. Yoshino, Breakdown study based on direct in situ observation of inner surfaces of an rf accelerating cavity during a high-gradient test, Phys. Rev. Accel. Beams 19, 102001 (2016).

[4] T. Abe et al., SuperKEKB design report. http://wwwsuperkekb.kek.jp/documents.html, 2014.

[5] T. Abe, T. Kageyama, Y. Takeuchi, H. Sakai, and K. Yoshino, RF accelerating structure for the positron damping ring of the SuperKEKB injector, in Proceedings of the 8th Annual Meeting of Particle Accelerator Society of Japan, TUPS131, 2011, http://www.pasj.jp/web_publish/ pasj8/proceedings/poster/TUPS131.pdf (Japanese).

[6] T. Abe, Y. Takeuchi, T. Kageyama, H. Sakai, and K. Yoshino, Development of $\mathrm{rf}$ accelerating cavity for the positron damping ring at SuperKEKB, in Proceedings of the 9th Annual Meeting of Particle Accelerator Society of Japan, THLR06, 2012, http://www.pasj.jp/web_publish/ pasj9/proceedings/PDF/THLR/THLR06.pdf (Japanese).

[7] T. Abe, Y. Takeuchi, T. Kageyama, H. Sakai, and K. Yoshino, High power testing of the rf accelerating cavity for the positron damping ring at SuperKEKB, in Proceedings of the 10th Annual Meeting of Particle Accelerator Society of Japan, SAP057, 2013, http://www.pasj.jp/ web_publish/pasj10/proceedings/PDF/SAP0/SAP057.pdf (Japanese).

[8] T. Abe, Y. Takeuchi, T. Kageyama, H. Sakai, and K. Yoshino, Test results on rf accelerating cavities for the positron damping ring at SuperKEKB, in Proceedings of the 11th Annual Meeting of Particle Accelerator Society of Japan, SAP050, 2014, http://www.pasj.jp/web_publish/ pasj2014/proceedings/PDF/SAP0/SAP050.pdf.

[9] The words "upstream" and "downstream" are used for convenience; no beam was injected into the cavity during the high-power test.

[10] https://ebajapan.jp/English/.

[11] https://ebajapan.jp/wp/wp-content/uploads/2018/04/pic_ originaltech-1024x599.png.

[12] The two lines were actually observed as one line spectrum by the hyperspectral camera.

[13] The spectrum data obtained at temperatures lower than $600^{\circ} \mathrm{C}$ had too much noise.

[14] http://www.ditect.co.jp/en/.

[15] The snapshots in the middle column are rotated by $180^{\circ}$ because the camera was upside down.

[16] R. Kersevan and M. Ady, Molflow+: a Monte-Carlo simulator package developed at CERN, https://cern.ch/ molflow/.

[17] M. Schmid, Vapor-pressure calculator, https://www.iap .tuwien.ac.at/www/surface/vapor_pressure/. Institute of Applied Physics, TU Wien.

[18] Thermo-Calc: Thermodynamics calculator, http://www .thermocalc.com/.

[19] https://www.cst.com/products/csts2. 